\title{
DART: A Simulation Code for Charged Particle Beams
}

\author{
R.C. White \\ UCID--21330-Rev, 1 \\ W.L. Barr \\ DE 89016697 \\ R.W. Moir
}

July 17,1989

\begin{abstract}
This paper presents a recently modified version of the 2-D code, DART, which csimulate the behavior of a beam of charged particles whose trajectories are determined by electric and magnetic fields. This code was originally used to design laboratory-scale and full-scale beam direct, converters. Since then, its utility has been expanded to allow more general applications. The simulation includes space charge, secondary electrons, and the ionization of neutral gas. A beam can contain up to nine superimposed beamlets of different energy and species. The calculation of energy conversion efficiency and the method of specifying the electrode geometry are described. Basic procedures for using the code are given, and sample input and output files are shown.
\end{abstract}

\section{DISCLAIMER}

This report was prepared as an accoupt of watk sponsored b

Government. Neither the United States Government nor any an agency of the United States employes, makes any warranty, express or implied, or assury agency thereof, nor any of their process disclosed acy, completeness, or usefulness of anyumes any legal liability or responsjence herein to any specific com that its use would not infringe privat, apparatus, product, or manufacturer, or otherwise commercial product. process, or service by trowned rights. Refermendation, or favoring by the not necessafily constitute or imply its te name, trademart. and opinions of aung by the United States Government or imply its endorsement, recomUnited States Government ar any herein do nol necessarily state or thereof. The views

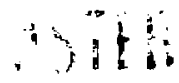




\section{Contents}

1 Introduction $\quad 4$

2 Running DART $\quad$ 5

3 Input Parameters 6

3.1 Parameters contained in Namelist /nl1/ . . . . . . . . . . . . 6

3.2 Parameters Contained in Namelist /nl2/ . . . . . . . . . . . . g

3.3 Parameters Contained in Namelist $/ \mathrm{nl3} / \ldots \ldots \ldots$. . . . . . . . . . . . . . . . . . . . .

3.4 Parameters Contained in Namelist /nl4/ . . . . . . . . . . . . 13

3.5 Parameters Contained in Namelist $/$ nl5/ . . . . . . . . . . . . 14

4 Input ard Output Files $\quad 15$

4.1 Input File DARTIN ........................ 15

4.2 Ouput File DARTOUT ...................... 16

5 What DART Does $\quad 19$

5.1 Solving the Equation of Motion ... . . . . . . . . . . . . . 19

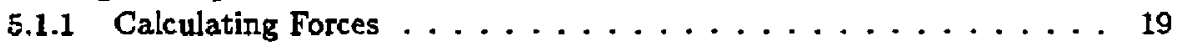

5.2 Space Charge Calculations . . . . . . . . . . . . . . . . . 20

5.3 Boundaries: Transparent, Fixed, Symmetry . . . . . . . . . . . 20

5.4 Starting Beams at Various Angles . . . . . . . . . . . . . . 21

5.5 Determining End Points and Efficiencies .............. 22

5.5.1 Conditions for Terminating of Trajectory Calculations . . . . . . . 22

5.5.2 Efficiency Calculations and Energy Conversion Check . . . . . . 22

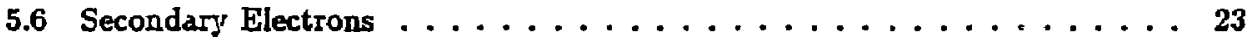

5.7 Cold Ions ............................ 23

6 Major DART Subroutines

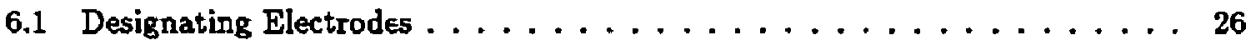

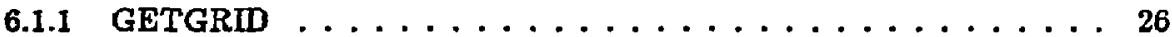

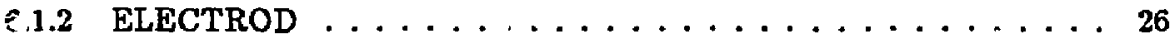

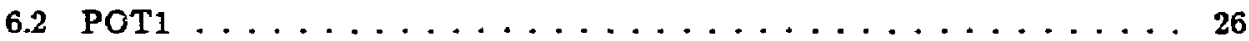

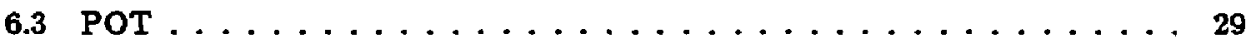

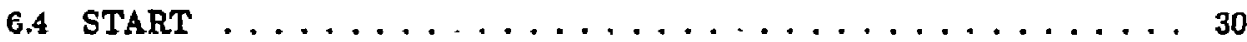

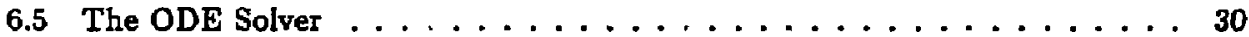

6.5.1 DRIVESA, STIFFA, COSETTA, and FUNCT ......... 30

6.6 PHI and $\mathrm{LAG} 2 \ldots \ldots \ldots \ldots \ldots \ldots \ldots$

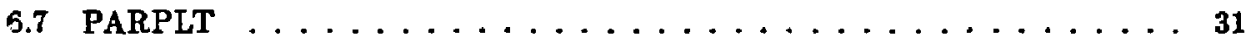

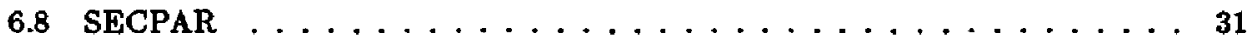

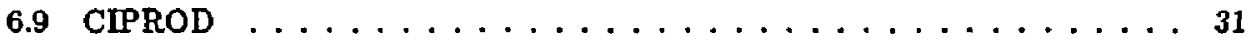

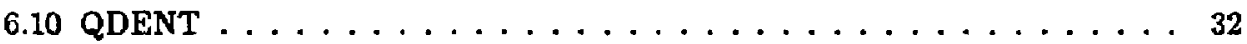

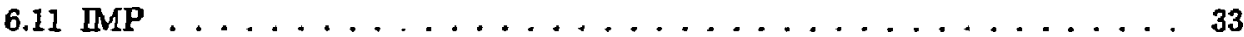

7 Modeling Electrons $\quad 35$ 
8 A Beam with Space Charge-Test Cage $\quad 36$

9 The Child-Langmuir Law-Test Case $\quad \cdot \quad 38$

10 A Test Case for Cold Ion Calculations $\quad 40$

11 Default Geometry $\quad 42$

$\begin{array}{ll}12 & \text { Summary }\end{array}$ 


\section{Introduction}

In 1971, a simulation code for a direct energy converter for use in fusion reactors was written by B.C. Howard in collaboration with W.L. Barr and R.W. Moir at Lawrence Livermore National Laboratory [1]. The code was developed for use in designing a direct energy converter system. The code has recently been revised to give it a wider variety of applications by allowing arbitrary placement of electrodes. This document describes the new (1987-88) version of DART. Although further modifications may be made, the operation of the code and the majority of input parameters will be preserved so that the utility of this document will not be severely jecpardized. The main changes made in this revision of this report were to allow the different beamlets to contain different species, as for example in the case of negative ion-based neutral beams that contain both negative and positive beamlets. Also, some of the discussions were expanded and some minor errors were corrected.

The basic calculations the code performs are as follows:

1. Read initial conditions and electrode data from the file, DARTIN.

2. Display the data and prompt the user for changes.

3. Construct internal electrode information from data.

4. Solve Laplace's equation for the vacuum potential.

5. Initialize the primary ion trajectories for a beamlet.

6. Calculate electric and magnetic forces, solve the equation of motion, record charge density data, and plot trajectories of charged particles for this beamlet.

7. Determine trajectory end points (impact locations) and energy conversion efficiencies for this beamlet.

8. Start secondary electrons (if ispar is not zero) from each ion trajectory impact point, with an energy-dependent emission coefficient.

9. Plot secondary electron trajectories (if ispar is not zero), and summarize secondary electron data.

10. Repeat the above steps for each beamlet.

11. Start cold ion trajectories from selected mesh points, weighted with the sum of production rates gathered from primary ion \$rajectories.

12. Plot cold ion trajectories (if requested by setting icold not zero), and summarize cold ion data, including total efficiencies.

13. Solve the Poisson equation to determine a new space charge potential table for the next iteration. 
14. Repeat the above steps starting at step 5 for itmax number of space charge iterations, and then terminate this run.

A "beam" consists of superimposed components, or "beamlets". Each beamlet contains a single species and a single original energy, starting position, width, current distribution, and orientation. Each beamlet is approximated by a finite set of trajectories that are spaced uniformly at the start. The trajectories are calculated by solving the equations of motion at each step. Both electric and magnetic fields may be included. Electric fields are calculated from a potential function whose values are obtained by interpolation in a twodimensional table. Initially, the vacuum potential is calculated. The most recent values of space charge are included in each iteration of the potential calculation. Charged particle trajectories and a new distribution of space charge are calculated for a specified number of trajectory iterations. The number of trajectory iterations is an input parameter called itmax. DART can continue with further trajectory and space charge iterations after the run is complete, by changing the parameter from isc $=0$ to isc $=1$.

Each ion trajectory is followed until it terminates either on an electrode or on a wall, and the efficiency of energy conversion is calculated. The tabulation of electric currents to the electrodes and walls provides data for evaluating the separate heat loads and erosion rates. After all beam components have run, DART computes the total efficiency of the energy conversion.

\section{Running DART}

Compilation and loading are necessary to obtain the executable version of DART. To compile and load the code on the Cray computer, one must use the CIVIC compiler and type:

$$
\text { civic } i=\operatorname{dartl} / t \boldsymbol{t}
$$

This step must br: redone if any changes are made to the source code, dartl.

We begin with a short description of the DART code, its input, output, and graphics. The necessary libraries are loaded automatically. These consis: if FORTLIB and GRAFL3. FORTLIB is the standard Fortran library on the Cray computers, while GRAFL3 is a collection of easy-to-use graphics modules based on the more sophisticated graphics library called GRAFLIB [2].

Running the code requires an input file called DARTIN. This file may be constructed or modified with your favorite editor. Editors supported on the Cray computers include TRIXGL and TEDI. (Examples of input files are provided in Sections 4, 8, 9, and 10.) Then simply type:

$$
\text { dart / } t v
$$

or

$$
\text { dart } i=<\text { filename }>/ t v
$$


if an input file, $<$ filename $>$, is to be used instead of the default "dartin". All units are SI, except that potentials are measured in kilovolts, energies in kilo-electron-volts, and gas pressure in Torr.

\section{Input Parameters}

All input parameters for this code are arranged in namelist blocks, allowing them to be grouped and displayed for user interaction with the DART code.

Initialization of these parameters is done with one input file, DARTIN. Variables not set in DARTIN will be equal to their default values, given in the sections which follow. Changes can be made to DART input parameters when the user is prompted by the DART code. These changes override the input file values for this run without changing DARTIN.

DARTIN contains namelist input with the variables listed in the sections which follow. A short description accompanies each variable along with its default value (shown in parentheses). The namelists are labeled by $/ \mathrm{nl} 1 /, / \mathrm{n} \mid 2 /, / \mathrm{nl3} /, / \mathrm{nl} / \mathrm{h}$, and $/ \mathrm{nl} 5 /$. Changes to the first two namelists can only be made in DARTIN, whereas the last three are written to the terminal, where changes can be made to them. Details of how to use DARTIN and how to input from the terminal are described in Section 4.

These lists of input parameters, presented in the order in which they are accepted by the DART code, are intended for use as an input parameter directory. Variables with names that begin with $i, k$, or $n$ are integers. All others are real numbers. The units of the input parameters are: $m, k V$, keV, radians, Torr.

\subsection{Parameters contained in Namelist /nl1/}

imin: The minimum $x$-grid index. This integer determines the location of the left wall. imin must be greater than or equal to zero (default: 0 ). For an example of this and the following parameters. See Fig. 1.

imin, imax: The minimum and maximum $x$-grid indices. These integers determine the locations of the left and right walls. They must satisfy the relation: $0 \leq i \min <$ imax $\leq$ 150. (default: 0,60 )

kmin, kmax: The minimum and maximum z-grid indices. These integers determine the locations of the lower and upper walls or symmetry planes. They must satisfy the relation: $0 \leq k m i n<k m a x \leq 100$. (default: 0,40 )

xmin, Xmax: Minimum and maximum values of $x$ cootdinate. These variables and imax and imin determine the grid spacing in the $x$ direction. imax is the maximum gridpoint value, with $i=i \max$ at $x=x \max$ and $i=i \min$ at $x=x \min$. (default: 0.0 and 0.6)

zmin, zmax: Minimum and maximum values of $z$ coordinate. These variables and $k$ max and $k \min$ determine the grid spacing in the $z$ direction. $k$ max is the maximum grid-point value, with $k=k \max$ at $z=z \max$ and $k=k \min$ at $z=z \min$. (default: 0.0 and 0.4 ) 


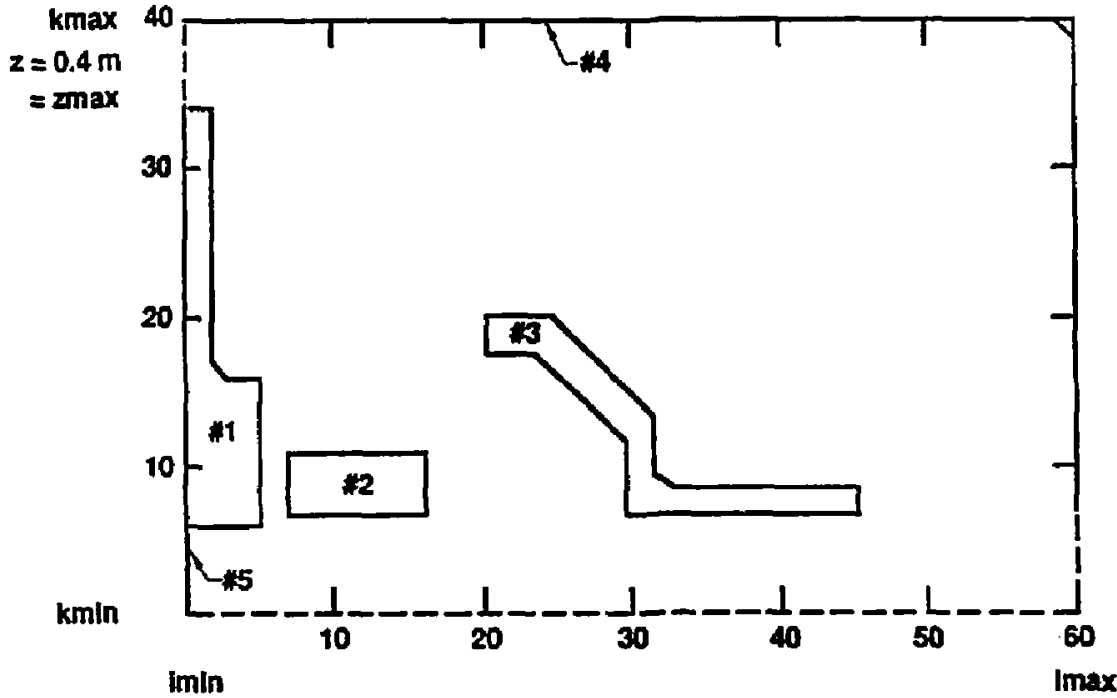

$$
\begin{aligned}
x & =0.6 \mathrm{~m} \\
& =x \max
\end{aligned}
$$

Figure 1: Diagram showing grid spacing and location of various input parameters for the default test case (see Section4.1). 
vref: The potential value at which ions are started. vref is used in efficiency calculations. It is also used to determine which potential contours will be solid (greater than vref) and which will be dashed (less than vref). (default: -100.0 )

isymp(1): $l l=1,2,3,4$ symmetry plane designator isymp(1) $=1$ implies a symmetry plane at bottom: $z=0$ isymp (2) $=1$ implies a symmetry plane at top: $z=z \max$ isymp $(3)=1$ implies a symmetry plane at left: $x=0$ isymp $(4)=1$ implies a symmetry plane at right: $x=x \max$ (default values: isymp $=1,0,0,0$ ).

\subsection{Parameters Contained in Namelist /nl2/}

eps1: Convergence parameter for over-relaxation. When a potential table is being calculated, consecutive iterations of the relaxation loop are compared. If the rms value of the difference between successive potential values for all points involved is less than epsi times a measure of convergence, the process is terminated. To get more accurate results, we can decrease the value of eps1, but the process will take more time to converge (default: 0.02 )

kppi: The z-grid index at which to output potential values as a check on relaxation convergence. Values are written to the output file DARTOUT at regular intervals of the $x$ coordinate. (default: 2)

iternm: Maximum number of iterations to perform for potential relaxation. This is a limit to avoid spending too much time in the space charge calculations. Typically the actual number of iterations should be less. If not, eps1 may be adjusted. The lists of sample potential values for each iteration given in DARTOUT are useful to check this calculation. (default: 150)

jelmod: Switch for modeling electrons. ielmod $=0$ indicates that no electrons are to be used. $i e l \bmod =1$ indicates that a Boltzmann distribution is to be used. ielmad = 2 clips the potential at a specified value. For details, see Section 7. (default: 1)

te: Electron temperature for use in Boltzmann distribution. See Section 7. (default: 0.01)

ine1 (ine2, ine3): The $x$-grid index where electron charge density begins. It is used in defining region one (two, three). In the default case, region one is near the suppressor, and regions two and three each include all space ocupied by the beams. Where regions overlap, the region with the highest number dominates. Figures 2 and 3 show examples. (default: $0,0,0$ )

ipe1 (ipe2, ipe3): The x-grid index where electron charge density is cut off. It is used in defining region one (two, three). In the default case, region one is near the suppressor, and regions two and three each include all space ocupied by the beams. Where regions overlap, the one with the highest number dominates. Figures 2 and 3 show examples. (default: 12, 60, 60) 


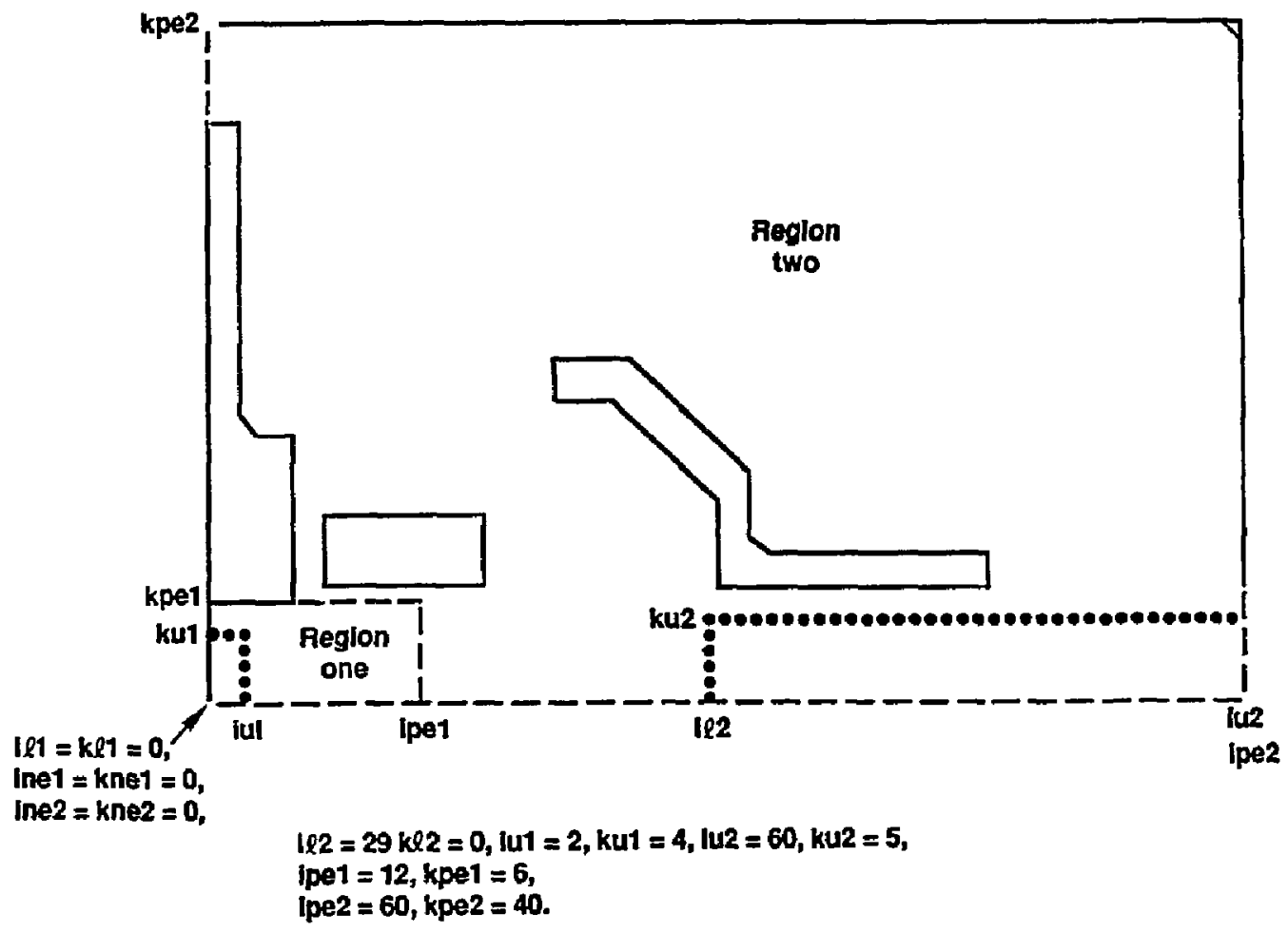

Figure 2: Limits defining electron distribution parameters for the default test case. Region enclosed in dashed lines represents region one. Dotted lines surround regions for average ion charge density calculations. See Section 7. 


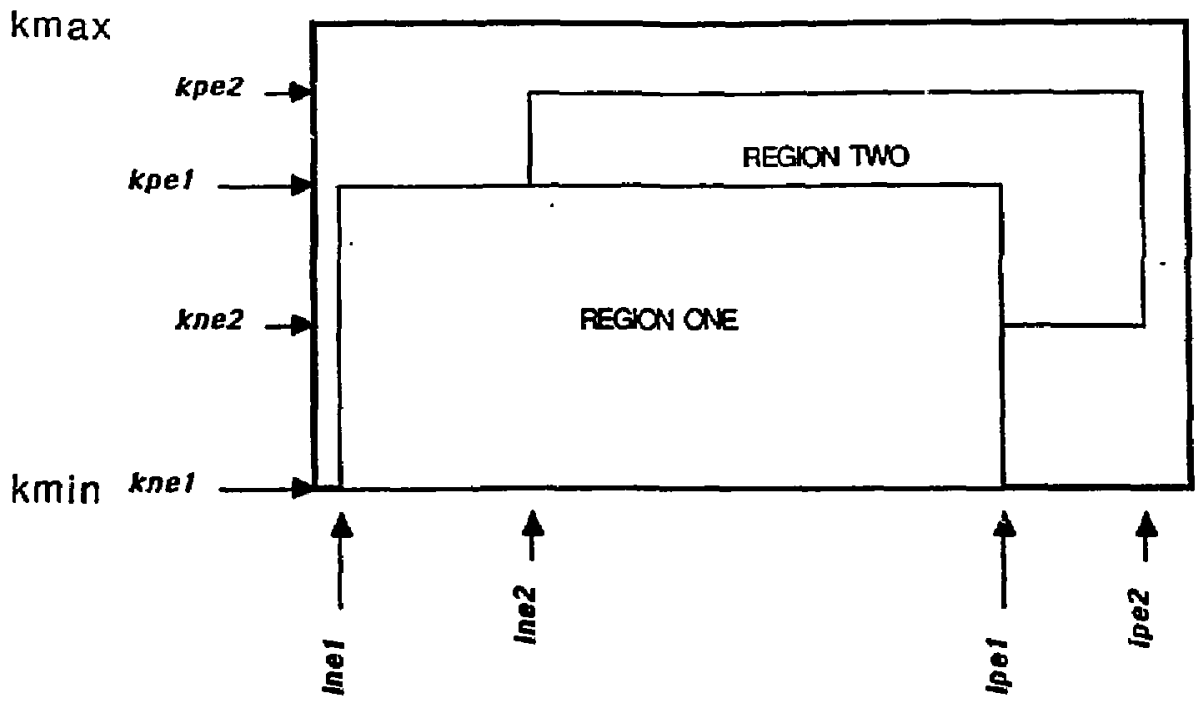

$\mathrm{kmax}$

$\mathrm{kmin}$
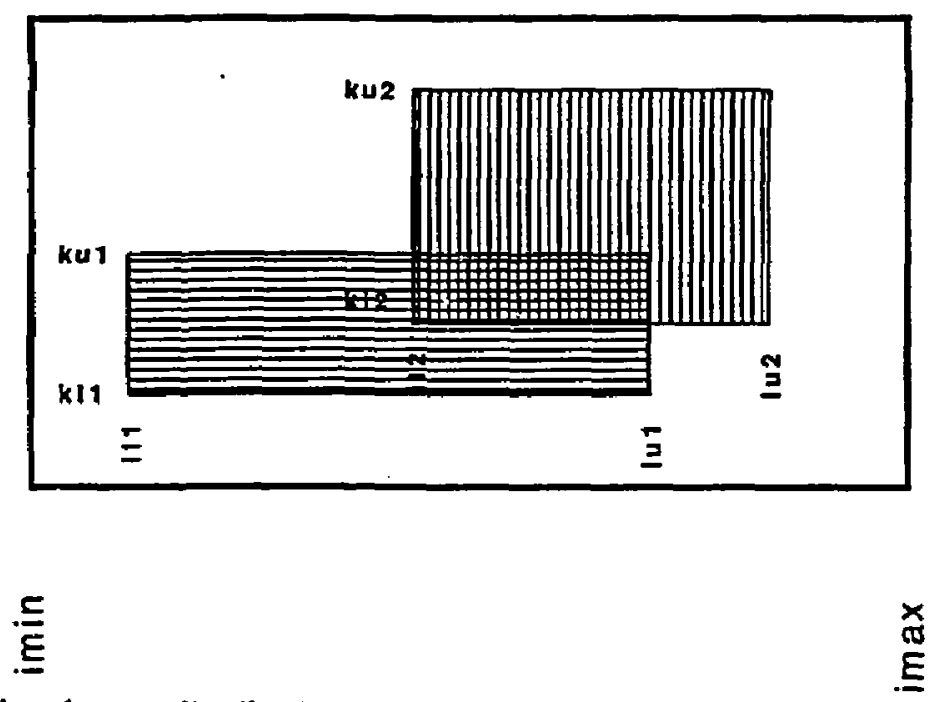

Figure 3: Limits defining electron distribution parameters. Regions one and two are defined in the top diagram. In the bottom diagram, we define the areas where average ion charge densities are calculated. Horizontal indicates area shading for use in region one; vertical shading, for use in region two. 
kne1 (kne2, kne3): The z-grid index where electron charge density begins, used to define region one (two, three). (defauit: 0 )

kpe1 (kpe2, kpe3): The $z$-grid index where electron charge density is cut off, thereby defining region one (two, three). (default: 6, 40, 40)

ill (il2, il3): Lower bound for the $x$-grid index for region one (two, three) where average ion charge density is calculated to reference the Boltzmann electron distribution in that region. See Section 7. (default: $0,29,29$ )

iul (iu2, ius): Upper bound for the I-grid index for region used to calculate average ion charge density. (default: $2,60,60$ )

kl1 (k12, kl3): Lower bound for the z-grid index for region used to calculate average ion charge density. (default: $0,0,0$ )

ku1 (ku2, ku3): Upper bound for the z-grid index for region used to calculate average ion charge density. (default: $4,5,5$ )

vg1 (vg2, vg3): Reference potential (or cut-off potential) for electron density distribution in region one (two, three). (default: $-100.0,0.0,0.0$ )

ichl: Switch to run Child-Langmuir law test case. This switch should ONLY be set to one when the sample input file shown in Section 9 is used. The generation of plots specific to this test case deems this necessary. (default: 0 )

\subsection{Parameters Contained in Namelist /nl3/}

beta: A damping factor used in the accumulation of charge density, sometimes needed to avoid overshooting the change in charge density from one iteration to the next. bet $2=1$. gives no damping. Otherwise, a fraction 1 - beta of the previous charge density is added to beta times the present space charge. (default: 1.)

iz(i): Charge state ( + or - ) of particles in the $i^{\text {th }}$ beamlet. The charge on the particles is equal to $i z(i)$ times $1.3 \times 10^{-19}$ C. (default: $1,1,1,0,0,0,0,0$ )

$n a(i)$ : Mass number of particles in the $i^{\text {th }}$ beamlet. The mass of the particles is equal to $n a(i)$ times $1.67 \times 10^{-17} \mathrm{~kg}$. For a beam of deuterium ions for example, set na(i) = 2 and $i z(i)=2,2,2,0,0,0,0,0)$

hbeg, hmin, hmax: Beginning, minimum, and maximum time step sizes. hmin is the minimum time step allowed. hmax should be chosen to ensure accuracy and to save time. If hmar is initially set to zero, it will be optimized based on beam energy. $s s d t$ must be greater than $h$ max to ensure that all snapshot times accumulate charge density. See Section 6.10. (defaults: $1 . \times 10^{-11}, 5 . \times 10^{-15}, 0.0$ )

tbeg: Start time. (default: 0 ) 


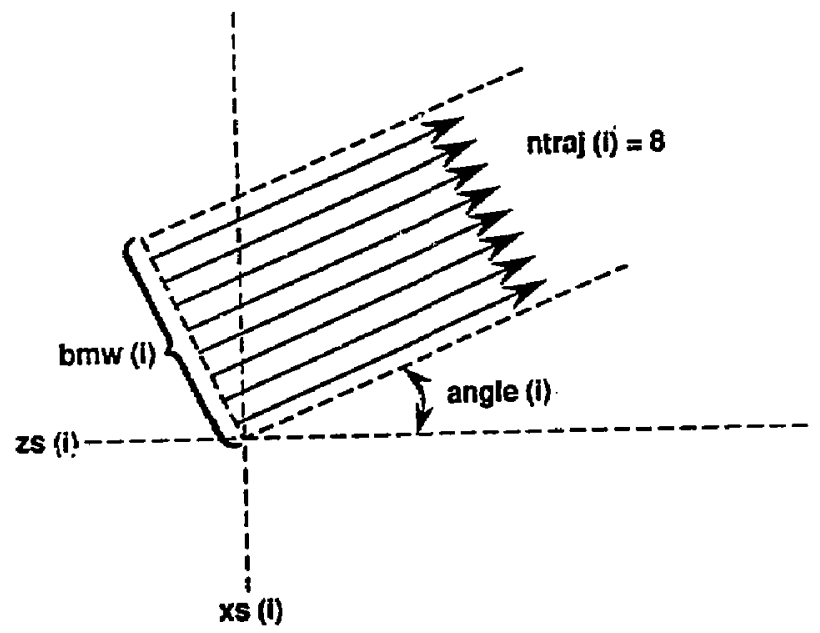

Figure 4: Illustration of starting conditions (xs, zs, and angle) fir a beamlet.

tmax: Cutoff time for trajectories in seconds. This limit avoids excess time following tortuous trajectories. (default: $1 . \times 10^{-05}$ )

eps, freps, errmx: Error parameters for integrator. For definitions, see Section 5.5.2. (defaults: $5 . \times 10^{-05}, 0.2,0.01$ )

ssdt: Snapshot time interval for charge density calculations. At each snapshot time, charge density is allocated to the four corners of the surrounding grid cell. ssdt should be chosen greater than the maximum allowed step size. See Section 6.10. (default: $1.5 \times 10^{-9}$ )

xs(i): Starting I coordinate value for ith beam component. Eight different beam components are allowed in the present version of the DART code. The total number of components is determined by the energy(i) array. See Fig. 4 for a deseription of this and the following input variables. (default: $0 ., 0 ., 0 ., 0 ., 0 ., 0 ., 0 ., 0$ )

zs(i): Starting $z$ coordinate value for $i$ th beam component. (default: $0,0,0,0,0,0,0,0$ ) bmw(i): Full beam width (m) for $2^{\text {th }}$ beam component. (default: $0.06,0.06,0.06,0 ., 0$, 0., D., 0.)

angle(i): Starting angle (radians) for $i^{\text {th }}$ beam component relative to the $(x, y)$ plane. (default: 0., 0., 0., 0., 0., 0., 0.,0.)

energy (i): Beam energy (keV) for $i^{\text {th }}$ beamlet. When the first value of energy(i) equal to zero is encountered, the code assumes that is the last one. To start a beamlet with zero energy, choose an energy that is negligibly small but not zero. Eight energies are allowed. (default: 100., 50., 33., 0., 0., 0., 0., 0.) 
ntraj(i): Total number of trajectories to calculate for the ith beam component. See Section 6.4. (default: 20, 20, 20,0,0,0,0,0)

numpl: Maximum number of trajectories per plot. Choosing a smaller value of numpl will help save memory: plotting arrays are dynamically dimensioned with numpl. (default: 20)

z0(i): Scale factor, $z_{0}$, in the Gaussian distribution for $i^{\text {th }}$ beamlet:

$$
f=f_{0} \exp \left\{-\left(\frac{r-z_{1}}{z_{0}}\right)^{2}\right\} .
$$

If $z 0(i)=0$, a flat-top distribution is used; ions are distributed uniformly across the beam with the same partial current: $I_{p}=\operatorname{tcrnt}(i) /$ ntraj $(i)$. See Section 6.4 for details. (default: $0.0632,0.0632,0.0632,0 ., 0 ., 0 ., 0 ., 0$. )

z1(i): $z_{1}$ for the ith beam component in the expression above. (default: $0 ., 0 ., 0 ., 0 ., 0$, 0., 0., 0.)

tcrnt(i): Total current density (in $\mathbf{A} / \mathbf{m}$ ) for each beam energy (i). If the beam is adjacent to a symmetry plane, enter only half the total current. For a description of use, see Section 6.4. (default: 9.7, 1.05, 0.455, 0., 0., 0., 0., 0.)

\subsection{Parameters Contained in Namelist /nl4/}

wcold: Starting energy of cold ions. (default: $1 . \times 10^{-04}$ )

press: Gas pressure (in Torr) for cold ion production. (defăult: $2 . \times 10^{-01}$ )

nctr: Maximum number of cold ions to be followed. For description, see Section 6.9. (default: 40)

id: Identifier for cold ion cross-section calculations. Values of $i d$ correspond to different combinations of ions/background gas: id $=1$ means $\mathrm{He}^{+} / \mathrm{He} ; i d=2$ means $\mathrm{He}^{+} / \mathrm{H}_{2}$; id $=3$ means $\mathrm{H}^{+} / \mathrm{H}_{2}, \mathrm{D}^{+} / \mathrm{D}_{2}$, or $\mathrm{T}^{+} / \mathrm{T}_{2}$. (default: 3 )

iprint: Number of times to skip between prints to output file. If iprint is nonzero, the code prints time, position, velocity, kinetic energy, and total energy. No print if 0 . (default: 0)

isc: Switch to indicate continuation of space charge trajectory iterations. When isc $=0$, DART starts with a vacuum potential calculation. When isc $=1$, the potential table from a previous run is read from ihe file POTAB. An error will result if isc $=1$ and POTAB does not exist. (default: 0 )

iged: Switch to calculate charge density table when set to 1 . When igcd $=\mathrm{I}$, QDTAB is used or is created. When igcd $=0$, charge density is not accumulated. Must have $i g e d=0$ whenever $i c o l d \neq 0$. (default: 1 ) 
jqtab: Switch to accumulate charges (1) or create a new table (0) for charge densities. DART adds to QDTAB when igtab $=1$ and creates QDTAB when iqtab $=0$. This switch is only used if igcd $=1$ (default: 0 )

ispar: Switch to include secondary electrons. If set to 1 , trajectories originate from impact locations on electrodes. For more details, see Section 5.6. (default: 0)

icold: Switch to include cold ions. Cold ions are included when icold $=1$. (default: 0 )

icsc: Switch to calculate space charge contribution from cold ions. Cold ion space charge is included when icse $=1$. (default: 0 )

itmax: Maximum number of space charge trajectory iterations. (default: 1)

xlev: Spacing (kV) for potential contours. (default: 5)

sesf: Scaling factor for secondary electron emission coefficient. ses $f$ multiplies $\gamma$, to allow for particular surface conditions. See Section 5.6. (default: 1.0)

pmpr: Percentage of cold ion maximum production rate, below which DART skips the cold ion calculation at that mesh point. (If cipror $(i, k)>a m x p r * p m p r$, DART starts a cold ion trajectory, where amxpr is the maximum rate calculated for the entire grid and cipror $(i, k)$ is the pruduction rate for the grid point.) See Section 6.9. (default: 0.1)

\subsection{Parameters Contained in Namelist /nl5/}

Electrodes (numel) can number between one and nine. A fixed boundary is input with the electrodes (boundaries are explained in Section 5.3). The potential of the nth electrode (or wall) is assigned with the array value, vinput(n). Electrodes are input with arrays $i x$ and $k z$. All electrode data are contained in namelist /n]5/.

$\mathrm{ix}(\mathrm{n}, \mathrm{j})$ : Array containing the $x$-grid values for the $n$th electrode. The $j$ th value is the $x$ location of the $j$ th point. The last entry must be " -1 ", which signifies the end of the data string for this electrode. The code automatically reconnects the last point to the first, so for an electrode with four corners, $j$ ranges from 1 to 5 , with $i x(n, 5)=-1$.

$k z(n, j)$ : Array containing the $z$-grid values for the $n$th electrode. The $j$ th value is the $z$ location of the $j$ th point. The same rules apply as in the $i x$ array. Remember that. the last entry must be " -1 ".

numel: The total number of electrodes (incuding walls). In the test case described Section 4, we set numel $=5$. (default: 1)

vinput(n): The potential $(\mathrm{kV})$ assigned to the $n$th electrode. (default: $0 ., 0 ., 0 ., 0 ., 0$, $0 ., 0 ., 0 ., 0$. 
The first two namelists are intended to contain those variables that are least likely to be changed from one DART run to the next. The last three namelists are displayed to the terminal and the user is prompted for changes. Changes made from the terminal in this way affect only the present run and are not saved.

\section{Input and Output Files}

\subsection{Input File DARTIN}

DARTIN is the input file needed to run DART. It contains each of the namelists mentioned in Section 3. The simplest input file will run the default case:

\section{Sendnl1}

Sendnl2

Sendnl3

Sendnl4

$$
\begin{aligned}
& (i x(1, j), j=1,7)=005522-1 \\
& (k z(1, j), j=1,7)=3466161634-1 \\
& (i x(2, j), j=1,5)=771616-1 \\
& (k z(2, j), j=1,5)=117711-1 \\
& (i x(3, j), j=1,11)=20202329294545313124-1 \\
& (k z(3, j), j=1,11)=2018181277991320-1 \\
& (i x(4, j), j=1,5)=0606060-1 \\
& (k z(4, j), j=1,5)=4040640-1 \\
& (i x(5, j), j=1,3)=00-1 \\
& (k z(5, j), j=1,3)=50-1 \\
& \text { numel }=5 \\
& \text { vinput }=-100 .-140 .-10.0 .-100 . \\
& \text { Sendnl5 }
\end{aligned}
$$

Statements with dollar signs (e.g., Sendn/3) indicate the end of a namelist block (/n13/ in this case). The dollar sign says that you are through listing data for that namelist and are ready to begin listing data for the next namelist (in this case, /nl4/).

This default case is one space charge iteration with three different ion energies in the geometry designed for the neutral beams in the Tore-Supra experiment [3]. The graphics output generated by the DART code is shown in Figs. 5-7. 
Because the efault values for the electrode arrays are zero, all electrodes must be specified in DARTIN.

After DART reads from DARTIN, it writes the namelists containing input parameters to the terminal. Then it writes,

Make changes to this namelist here (end with a S):

You may then adjust any variable in the list to new values. Type them as they appear in the list, separating them with spaces. Carriage returns are ignored. When done, type "\$" and a carriage return. If you don't want to change anything, just type a $\$$ ", and press return.

For example, after the first namelist is displayed, and you are prompted with the above message, you can type:

$$
\text { vref }=0 . \$
$$

If you want to add an additional electrode from the terminal, set numel to the new value (add 1) and put the last elements of $i x$ and $k z$ to -1 , as described in Section 3.

If you entered data as specified but are unsure that DART is running, type "ctrlE s" and return. If you see

$$
\text { R.CV + darta, }
$$

DART is waiting for more input. Type " $\$$ " and return.

\subsection{Ouput File DARTOUT}

DARTOUT is an output fle generated by DART. It is intended mainly for diagnostic purposes, since the graphics file (below) contains most of the important results from the run. DARTOUT first lists the complete contents of the five namelists. Next is the nelec array, which shows a crude picture of the electrodes by showing zeros where there are no electrodes, ones where electrode one should appear, twos for electrode two, and so on up to a maximum of nine electrodes. DARTOUT then lists values of the vacuum potential at five selected points to show the progress of the relaxation process. This is followed with a table of the $x$ and $z$ coordinates of the mesh points. Next is a table of the potential at each mesh point. The potential table is followed by a summary of each of the trajectories, including initial and final positions and velocities, and energy check data. It then lists summaries for secondary electrons and cold ions, if they were included in the run. Then it starts over, listing potential values for the next space charge calculation. DARTOUT will be very long if many space charge iterations are performed (i.e., if itmax is large), so you may not want to print the entire file.

DART also produces an FR80 graphics [2] file that contains input data, the nelec table, described in the DARTOUT description, contours of vacuum and each space charge iteration of the potential, and a plot of the trajectories. The computer-generated pictures in Figs. 5-7 are examples of frames from this file. This graphics file also displays summary tables of primary trajectories, secondary electron trajectories, and cold ion trajectories. At each stage the current collected at each electrode is given, as is the total current from all particles for each electrode. 


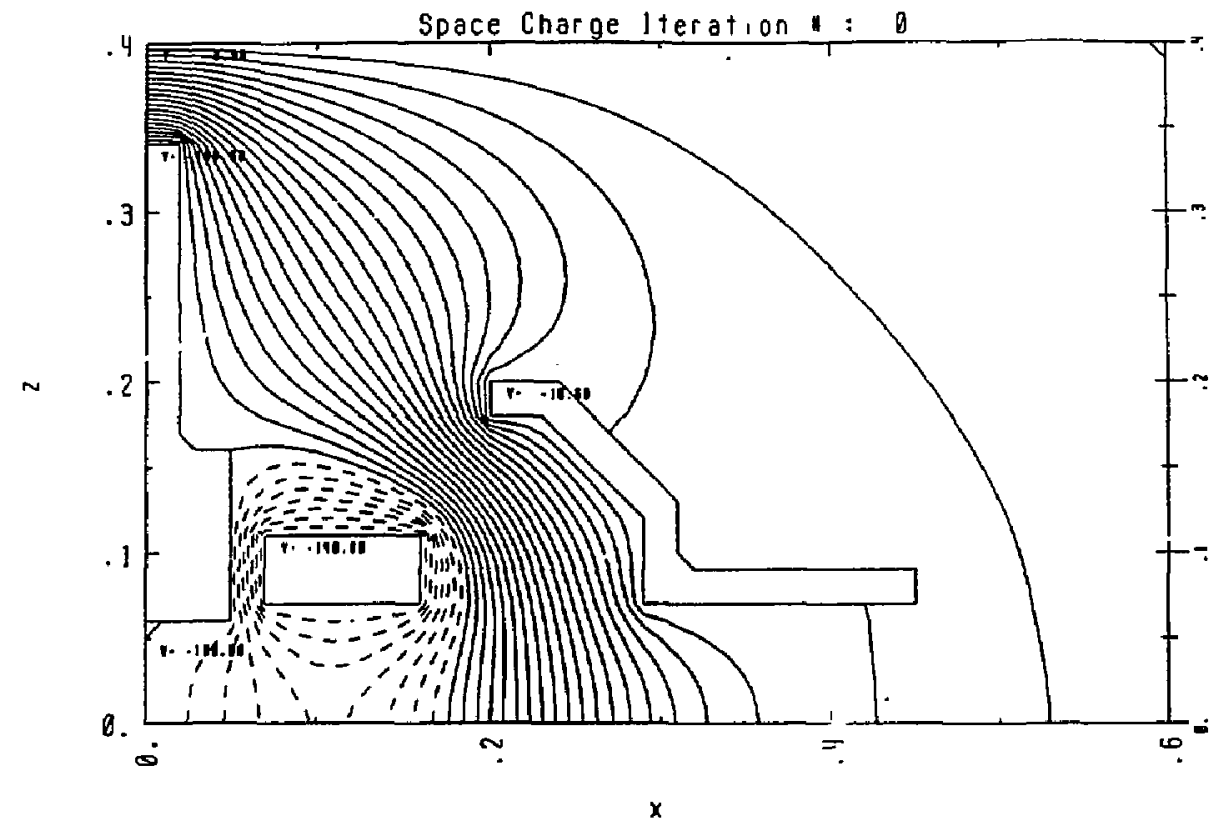

Figure 5: Vacuum potential contours for a direct converter design as generated by DART with default values.

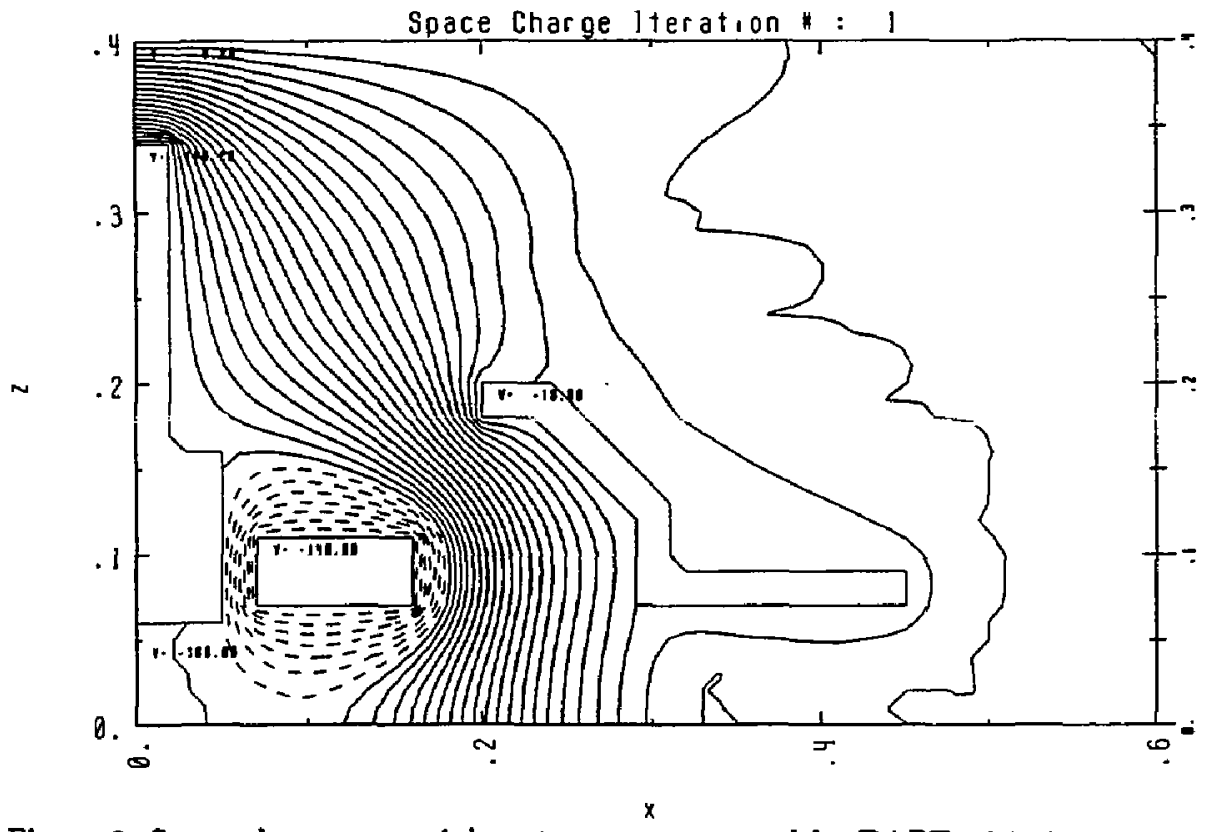

Figure 6: Space charge potential contours as generated by DART with detault values. 
(a)

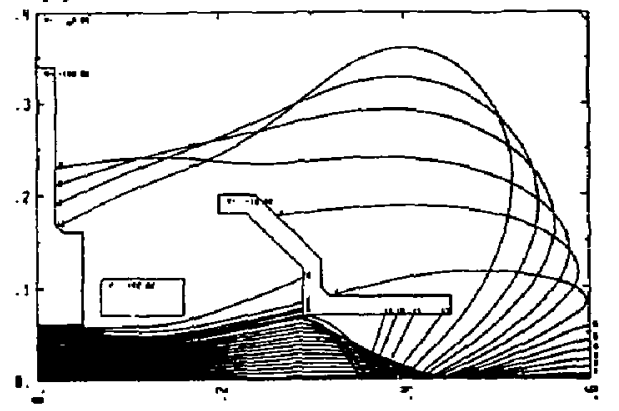

(b)

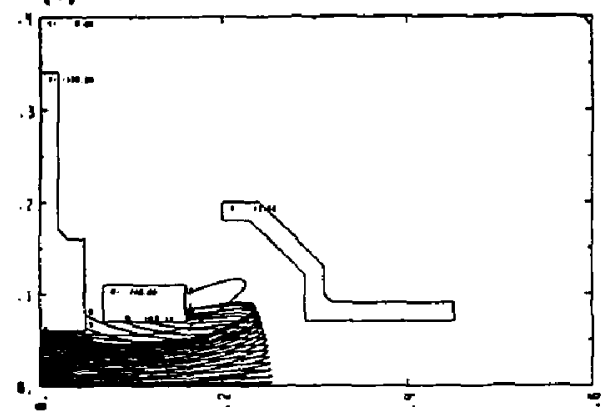

(c)

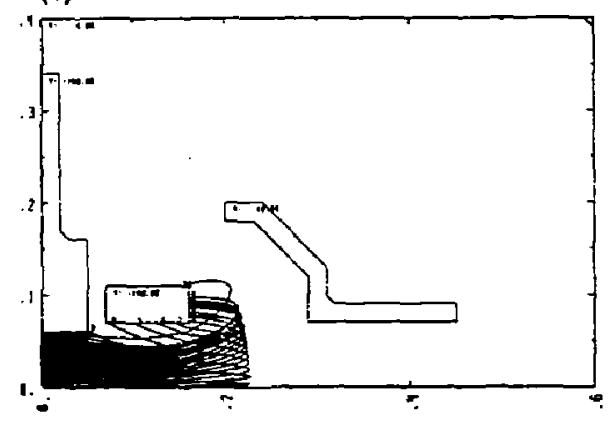

(d)

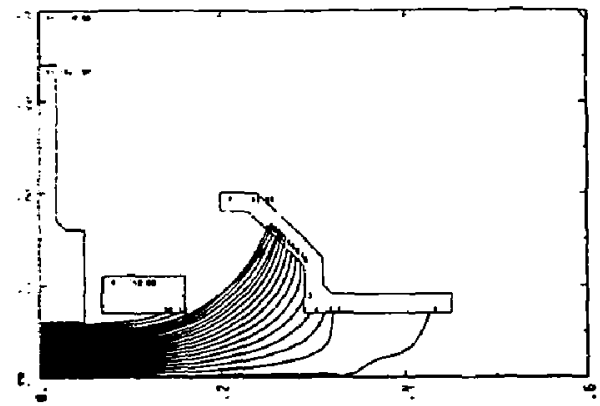

(e)

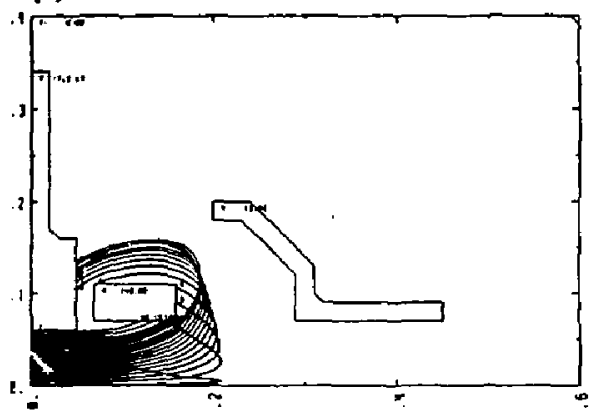

(i)

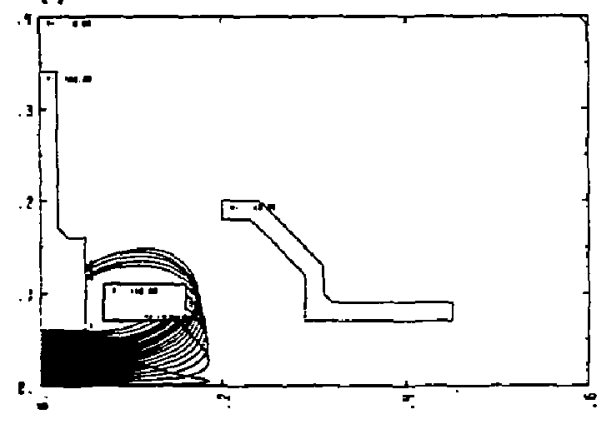

Figure 7: Ion trajectories as generated by DART with default values. (a-c) Trajectories in the vacuum potential; $(d-f)$ resulting trajectories in the first space charge iterated potential. 
The FR80 file always begins with "f3..." and the first one will be called "f3dart0x". The third character is incremented alphabetically as newer files are generated. The FR80 file can be printed at the default site (i.e., site=lll) by typing,

$$
\text { netplot f3dart0x / } t v
$$

With DART input and output described, we are now ready to discuss the internal workings of the DART code itself.

\section{What DART Does}

This section describes the mechanics of the DART code. We begin by discussing the equations of motion, and then we describe trajectory calculations with space cha-ge. Finally, we describe the optional computation of secondary electron and cold ion trajectories.

\subsection{Solving the Equation of Motion}

The second-order differential equation describing charged particle motion in an electromagnetic field,

$$
q \vec{E}+q(\vec{v} \times \vec{B})=m \vec{r},
$$

is decomposed into a set of first-order differential equations of the form,

$$
\frac{d}{d t} Y_{i}=F_{i} .
$$

By setting

$$
\begin{array}{ll}
Y_{1}=x & Y_{4}=\dot{x} \\
Y_{2}=y & Y_{5}=\dot{y} \\
Y_{3}=z & Y_{6}=\dot{z},
\end{array}
$$

we obtain

$$
\begin{array}{ll}
F_{1}=Y_{4} & F_{4}=\frac{q}{m} E_{z}+\frac{q}{m}\left(\dot{y} B_{z}-\dot{z} B_{y}\right) \\
F_{2}=Y_{5} & F_{5}=\frac{q}{m} E_{y}+\frac{q}{m}\left(\dot{z} B_{z}-\dot{x} B_{x}\right) \\
F_{3}=Y_{6} & F_{6}=\frac{q}{m} E_{x}+\frac{q}{m}\left(\dot{x} B_{v}-\dot{y} B_{x}\right) .
\end{array}
$$

The Adams method used to solve these equations numerically is described briefly in Section 6.5.

\subsubsection{Calculating Forces}

The electric field that a particle encounters is given by

$$
\vec{E}=-\nabla \phi .
$$

By the principle of superposition, each component is treated independently. The potential $\phi$ is determined from Laplace's equation to get the vacuum potential and by Poisson's equation to get the potential with space charge. 
The two-dimensional array igeom consists of ones and zeros. It determines the electrode locations and is also used in the potential calculation. The relaxation algorithm is used to calculate potential when igeom $(i, k)=0$, and the potential at the $(i, k)$ location is fixed when igeom $(i, k)=1$. The relaxation scheme is described in Section 6.2.

If a boundary is indicated by maximum or minimum values of coordinate arrays and no wall is specified, the relaxation algorithm assumes reflection symmetry. DART accomplishes this simply by assigning values just outside the boundary to be equal to the values just inside; values on the boundary are calculated by relaxation. Boundaries are described further in Section 5.3.

\subsection{Space Charge Calculations}

Space charge effects are caiculated in two steps. First, the charge density table is produced. Then, the method of relaxation is used to solve Poisson's equation and determine the new potential table. The number of trajectory iterations to be done in a given run is determined by the input parameter itmax. If further iterations are required to get a converged solution, you can run DART again with isc $=1$. The code then reads the potential table generated in the last iteration from disk file POTAB, which was created during the previous run.

For details on charge density calculation, see Section 6.10, and for details on the potential calculations, see Sections 6.2 and 6.3 .

\subsection{Boundaries: Transparent, Fixed, Symmetry}

The DART code has three kinds of boundaries. To distinguish them, we call them fixed potential boundaries, symmetry planes, or transparent boundaries.

The default is a transparent boundary. Such a boundary represents the end of a device without a wall. Where particles should continue beyond the edge of the field, we actually continue to integrate the equations of motion and to follow a trajectory for one cell beyond the boundary, a procedure that, allows charge density contributions to be calculated more precisely at the boundary. The plotted trajectory paths terminate at the boundary (as they do always), with the number of the trajectory displayed. The potential calculations are performed by use of symmetry. A fictitious row of values is set one cell length beyond the boundary. The values are set equal to potential values one cell length before the boundary, and the relaxation is performed up to and including the boundary.

A second type of boundary is the fixed potential boundary. By using electrode arrays $i x$ and $k z$, we can specify walls at a fixed potential. Only two points in these arrays (plus the all important -1) are necessary. See Sect. 3.5. The potential boundary values do not change during relaxation.

If you continue a wall around a corner without returning to that corner, the resulting calculation will be indistinguishable from that for an electrode occupying the corner. If ixed potential boundaries are input with two points only (plus the -1), this situation is avoided.

The third boundary is the symmetry plane. The default incorporates a symmetry plane at $z=0$. We use this boundary to save memory and potential calculation time by using the symmetry often found in such devices. To make a transparent boundary as on like the 


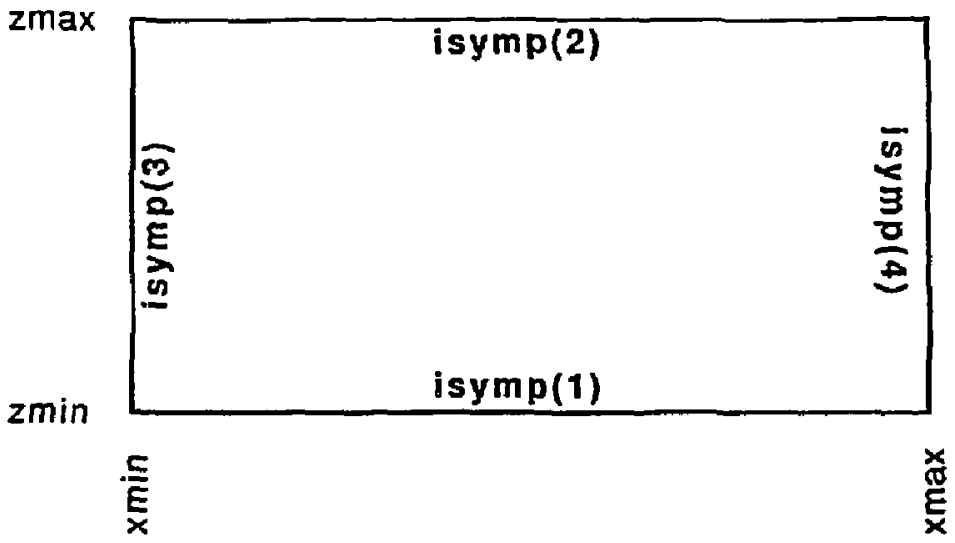

Figure 8: Symmetry plane specifiers at corresponding planes. When the specifier is set to 1 , its plane is a symmetry plane, and the specifier is set to 0 , its plane is a transparent plane.

other three walls, set isymp $(1)=0$. You also may add a symmetry plane at $z=z$ max by setting $i \operatorname{symp}(2)=1$. Symmetry planes at $x=0$ and $x=x$ max are constructed by setting isymp (3) $=0$, or isymp (4) $=0$, respectively. The symmetry plane arguments are deipicted in Fig. 8.

The DART code contains a variable called igrid. This variable indicates which electrode was impacted by a given trajectory. igrid is less than zero when a trajectory impacts a fixed boundary and is zero when a trajectory impacts a transparent boundary.

\subsection{Starting Beams at Various Angles}

The initial conditions for a beam component allow multiple beams from different starting positions and with different orientations. The beam components may also differ in energy and current profile. A beam component will start from within the following arrays:

- $x s(i)$ is the $x$ coordinate of the starting point for the $i$ th bearn component.

- $z s(i)$ is the $\mathrm{z}$ coordinate of the starting point for the $i$ th beam component.

- angle $(i)$ is the angle from the $(x, z)$ plane of the $i$ th beam component.

- $b m w(i)$ is the width of the ith beam component.

The energy and current are specified as follows:

- energy $(i)$ is the energy of the ith beam component.

- $z \mathrm{D}(i)$ and $z 1(i)$ are the current distribution parameters of the ith beam component. 
- tcrnt(i) is the total current (in $\mathbf{A} / \mathbf{m}$ ) of the ith beam component.

These input arrays are discussed in Section 3. The orientation for a given beam component is shown in Fig. 4. To start individual trajectories, set $n t r a j(i)=1$ and $b m w(i)=0$.

\subsection{Determining End Points and Efficiencies}

\subsubsection{Conditions for Terminating of Trajectory Calculations}

There are four conditions for terminating trajectory calculations:

1. If the value of the smallest allowable time step hmin is not small enough to maintain restriction on the local relative error, which is bounded by eps.

2. If the time used in integration exceeds the input parameter tmas.

3. If the trajectory exits beyond the boundaries specified by $x \min , x \max , z \min$, or zmax. The output parameter is igrid $=0$ in this case.

4. If the trajectory intersects an electrode (or a wall). Here igrid is the number of the impacted electrode. (It is negative for a wall.)

The most frequent (and most desired) form of termination is the last condition. Details on how an impact is detected are given in Section 6.11.

\subsubsection{Efficiency Calculations and Energy Conversion Check}

DART calculates the efficiency of a given ion trajectory from its initial and final potential energies. The efficiency is given by

$$
\eta=\frac{\phi_{f}-\phi_{0}}{W_{i}+\phi_{i}-\phi_{0}},
$$

where $\phi_{i}$ and $\phi_{f}$ are the initial and final potential energies in $\mathrm{kV} ; W_{i}$ is the initial kinetic energy in $\mathrm{keV} ;$ and $\phi_{0}$ is the reference potential input as vref, also in $\mathrm{kV}$.

The relative error in the total ion energy is given by the formula

$$
W_{\text {err }}=\left|\frac{\left(W_{f}+\phi_{f}\right)-\left(W_{i}+\phi_{i}\right)}{W_{i}+\phi_{i}}\right|,
$$

where the final inetic energy, $W_{f}$, is calculated from the final velecity components. If $W_{\mathrm{err}}$ is greater than errmx, then eps, the relative error test constant for the integrator STIFFA, is adjusted by multiplying it by freps. The trajectory is then recalculated. 


\subsection{Secondary Electrons}

Secondary electrons are included in a DART run if the switch ispar $=1$, and are not included if ispar $=0$. In subroutine SECPAR, secondary electron trajectories are started where an ion impacts an electrode, and they are initially directed back along the path of the incoming ion. Each trajectory carries a current equal to $\gamma$ times the current of the ion trajectory, where $\gamma$ is the secondary electron emission coefficient and is a function of impact energy. $\gamma$ is calculated in subroutine GAMMA; ses $f$ is a multiplier for $\gamma$ to adjust for different materials.

The true secondary electron emission coefficient is actually a function of many variables: charge and mass of the incident ion. target material (e.g., atomic number, atomic weight, electron structure), angle of incidence (and therefore the roughness of the electrode surface), and especially the chemical composition of the surface layer (e.g., gas layer, oxide layer). DART uses a simple expression that is a reasonable fit to the data for $\mathrm{D}^{+}$on clean Mo and that also fits the data for $\mathrm{D}^{+}$on $W$ and $\mathrm{He}^{+}$on $\mathrm{Mo}$ and $W$. In Fig. 9, DART's expression is superimposed on L.S. Hall's, Fig. 4 [7]. The high values are for gassy or oxide surfaces. The emission is thought to vary as the cosecant of the angle between the incident ion and the normal vector to the surface. We did not include this angle dependence because we did not have a reference that describes this effect quantitatively.

The formula used in subroutine GAMMA is

$$
\gamma \equiv \operatorname{ses} f \begin{cases}0.1 & \text { if } W \leq 0.1 \mathrm{keV} \\ 0.3 \sqrt{W} & \text { if } 0.1 \leq W \leq 100 \mathrm{keV} \\ 3.0 & \text { if } W \geq 100 \mathrm{keV}\end{cases}
$$

where the input parameter, ses $f$, can be used to adjust the secondary electron emission for particular surface conditions.

\subsection{Cold Ions}

An ion beam produces cold ions and electrons along its path by ionizing gas molectles, by dissociating gas molecules, and by charge exchange with gas molecules. The cold electrons contribute to the Maxwellian distribution of electrons that DART includes in the calculation of space charge. The cold ions not only contribute to space charge, but also deposit electrical current and heat on the electrodes where they are collected. The cross sections used by DART for determining the production rate of cold ions are shown in Figs. 10 and 11, both taken from C.F. Barnett et al. [4]. The user is cautioned that DART's cross sections may be inaccurate for $\mathrm{H}^{+}$energies below $0.5 \mathrm{keV}$ or above $2 \mathrm{MeV}$.

Cold ions can be started by setting, icold $=1$. No cold ion trajectories are calculated if icold $=0$. The array cipror is filled with values in subroutine QDENT, and cold ion trajectories are calculated in subroutine CIPROD. Details are described in Sections 6.10 and 6.9. The cold ion energy is given by the input parameter called wcold. The background gas pressure is press and the cross-section identifier is id (see Section 6.9).

Section 6.9 describes how cold ion trajectories are calculated, and Section 10 gives a simple test example for cold ions. 


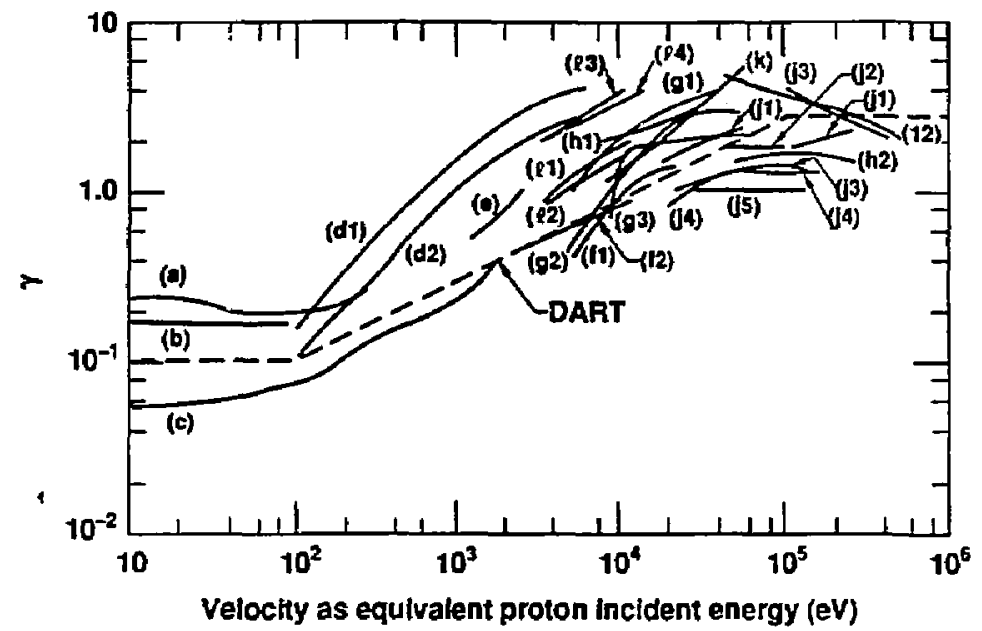
(a) $\mathrm{He}^{+}$on Mo
(b) $\mathrm{He}^{*}$ on (111) tace of $\mathrm{NI}$
(c) $\mathrm{H}^{*}$ on $\mathrm{MO}$
(d1) $\mathrm{H}^{+}$on $\mathrm{Cu}_{;}$(d2) $\mathrm{H}^{+}$on Mo and 302 stainless steel
(e) $\mathrm{He}^{+}$on $\mathrm{Mo}$
(t1) $\mathrm{He}^{+}$on $\mathrm{Mo}$; (t2) $\mathrm{H}^{*}$ on Mo
(g1) $\mathrm{H}^{*}$ on $\mathrm{Mo}$; (g2) $\mathrm{D}^{+}$on $\mathrm{Mo}^{\circ}(\mathrm{g3}) \mathrm{H}^{+}$on $\mathrm{Cu}$
(h1) $\mathrm{He}^{+}$on $\mathrm{W}_{;}$(h2) $\mathrm{H}^{+}$on $\mathrm{W}$
(i1) $\mathrm{H}^{*}$ on $\mathrm{Cu}$; (i2) $\mathrm{H}^{+}$on $\mathrm{Cu}$; (13) $\mathrm{H}^{+}$on $\mathrm{MO}$;
(i1) $\mathrm{H}^{+}$on $\mathrm{C}$; (j2) $\mathrm{H}^{+}$on Pt; (j3) $\mathrm{H}^{+}$on $\mathrm{Mo}$; (j4) $\mathrm{H}^{+}$on $\mathrm{Cu}$; (j5) $\mathrm{H}^{+}$on $\mathrm{TI}$
(k) $\mathrm{H}^{+}$on stainless stee!

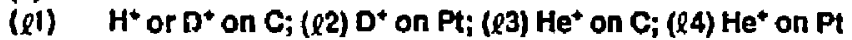

Figure 9: Secondary electron emission coefticient, $\gamma$, is shown as calculated in DART, superimposed on Hall's data. The table above is also from Reference [7]. 


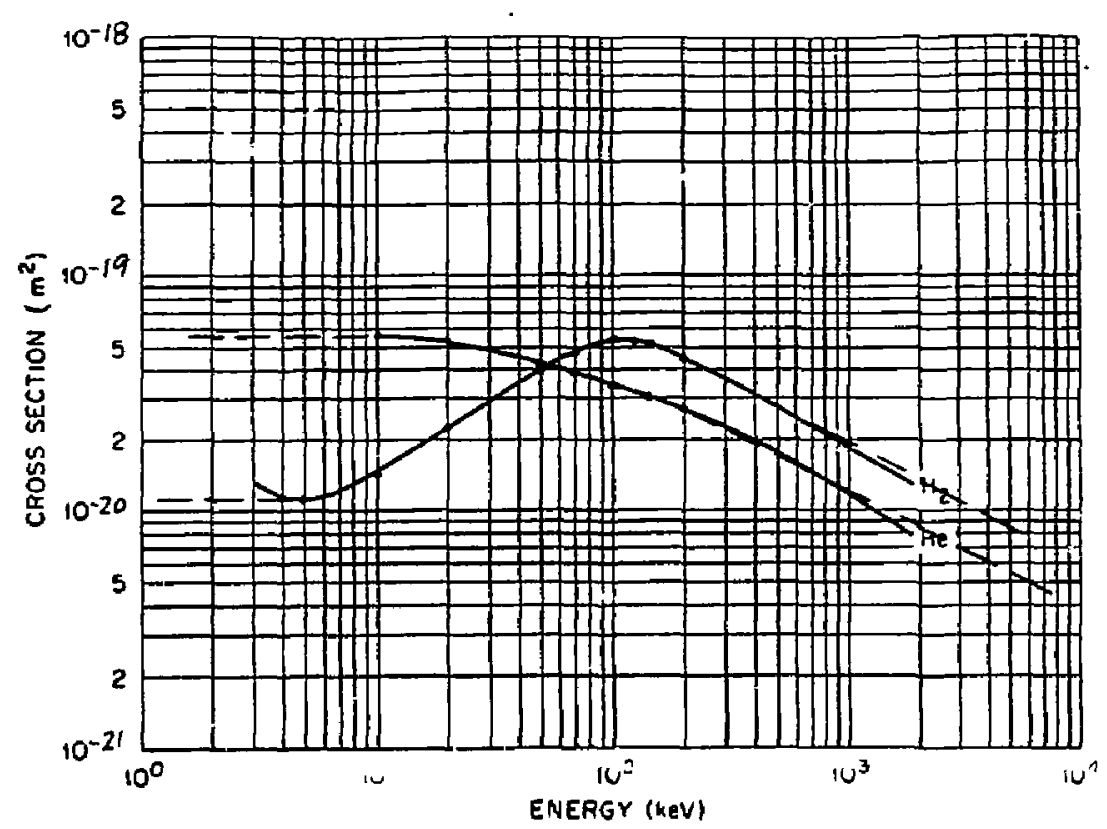

Figure 10: Cross section used to calculate production of positive ions by $\mathrm{He}^{+}$ions in $\mathrm{H}_{2}$ and He. DART uses the values shown by the dashed line. Data are from Reference [5], Fig. A.5.62.

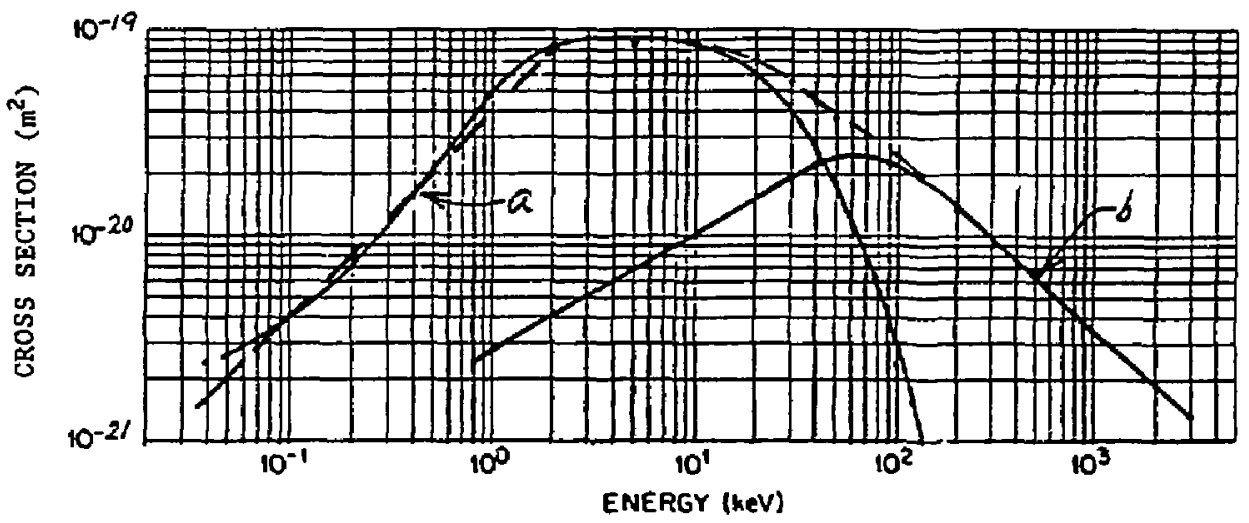

Figure 11: Dashed line shows total cross section used by DART to calculate the production of cold ions and electrons by $\mathrm{H}^{+}$in $\mathrm{H}_{2}$. Curves (a) and (b) are from Reference [7], Fig. A.4.5 (charge exchange) and Fig. A.5.7 (ionization by protons). For other isotopes of hydrogen, DART adjusts the energy scale to give the same velocity as $\mathrm{H}^{+}$. 


\section{Major DART Subroutines}

Each subroutine and related physics are described in the subsection beiring its name. For the most part subroutines are listed in the order in which DART's main subroutine calls them (Figs. 12, 13).

\subsection{Designating Electrodes}

\subsubsection{GETGRI}

The subroutine GETGRID calls the more specialized subroutine ELECTROD to set igeom, an array of ones and zeros indicating electrodes and spaces, respectively. vinput $(n)$ is used to assign the potential to the $n$th electrode region. GETGRID is called only once, when the code is initialized.

GETGRID calls ELECTROD numel times, where numel is the number of electrodes. Each electrode is then added to the array igeom. If any two electrodes overlap, an error message is printed to the terminal, and the code exits. First, however, igeom is printed to the output file, DARTOUT. To find the input error, look at DARTOUT with tedi or another editor, or type "type dartout." Overlapping electrodes are indicated by twos in the igeom array. Electrodes are allowed to lie next to one another, but they cannot share points.

\subsubsection{ELECTROD}

When the difference in consecutive $z$-grid coordinates from the array $k z(n, j)$ is negative, values to the right of the corresponding $i k(n, j)$ entries and the line interpolated between the $j$ th and $(j+1)$ th points have $a+1$ added. When the $z$ difference is positive, values to the right are changed by -1 . This basic principle is used to construct the electrodes. If the direction changes (left or right) while consistently moving down (or up), adding (or subtracting) twice is avoided.

Since the diagonal surface of an electrode will have the constant potential value of that electrode, inside corners only exist to the grid-scale size. In other words, consider a block of four grid points. An inside corner is represented by three ones and a zero. A diagonal boundary on an electrode is also represented by this configuration. Since there is no difference, we decided to treat particle trajectory impacts as diagonal boundary impacts. This decision eliminates the existence of true inside corners on electrodes, and is observable in the DART generated figures. For example, the upper right-hand corner of our default test case contains a small triangle. This triangle is an indicates that the wall input as electrode 4 bends from across the top down to the right side (see Fig. 5).

\subsection{POT1}

POT1 calculates the vacuum potentials for the geometry calculated by GETGRID. It uses the relaxauion algorithm, where no relaxation is done on the electrodes (i.e., where $\operatorname{igeom}(i, k)=1$ ). 
(a)
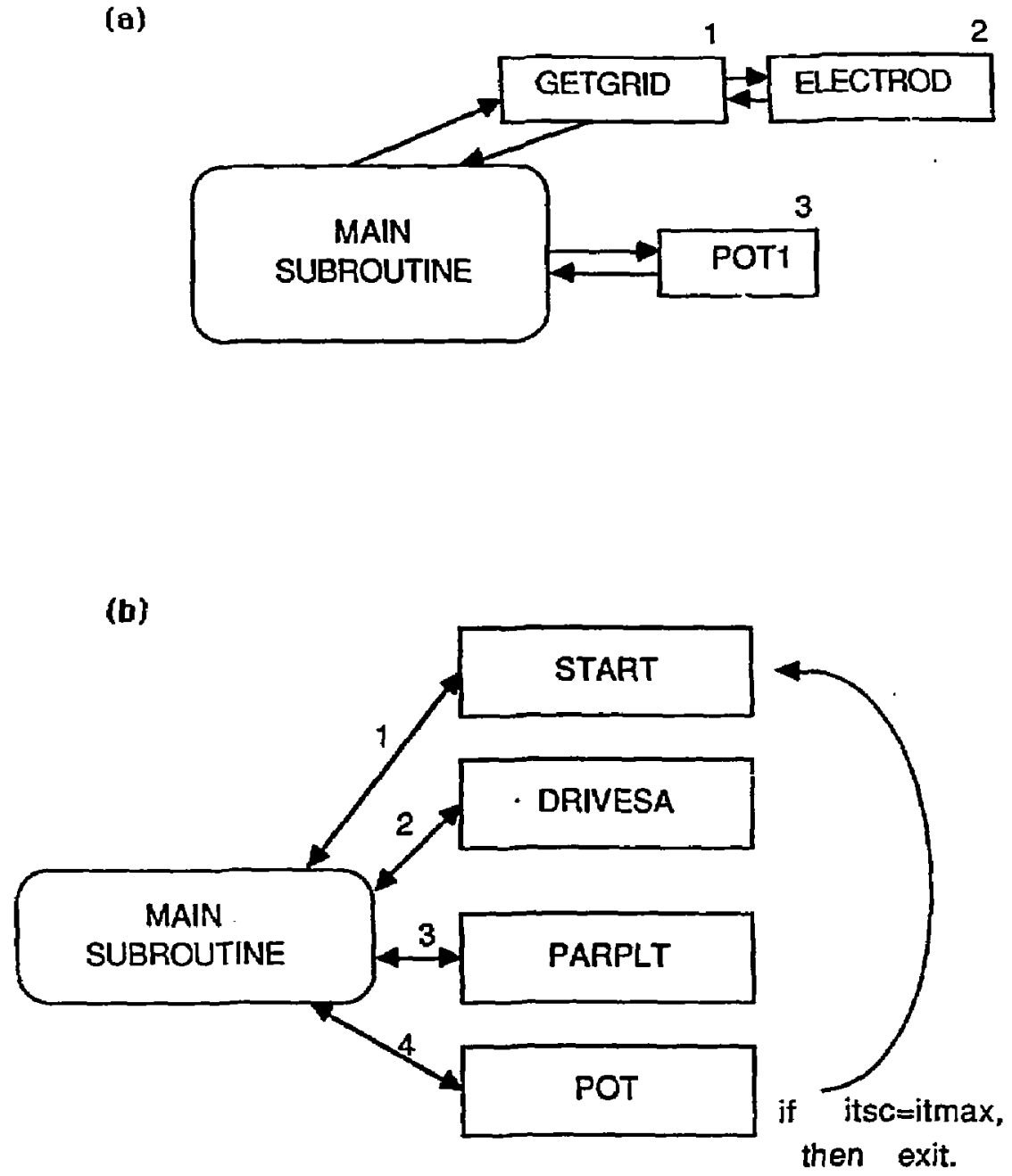

itsc: present space charge iteration number itmax: maximum space charge iteration number

Figure 12: Calling sequence and control for major DART subroutines. The main subroutine (a) calls the initializing subroutine in the order shown, then (b) calls four subroutines in a loop until all trajectory iterations are completed. 

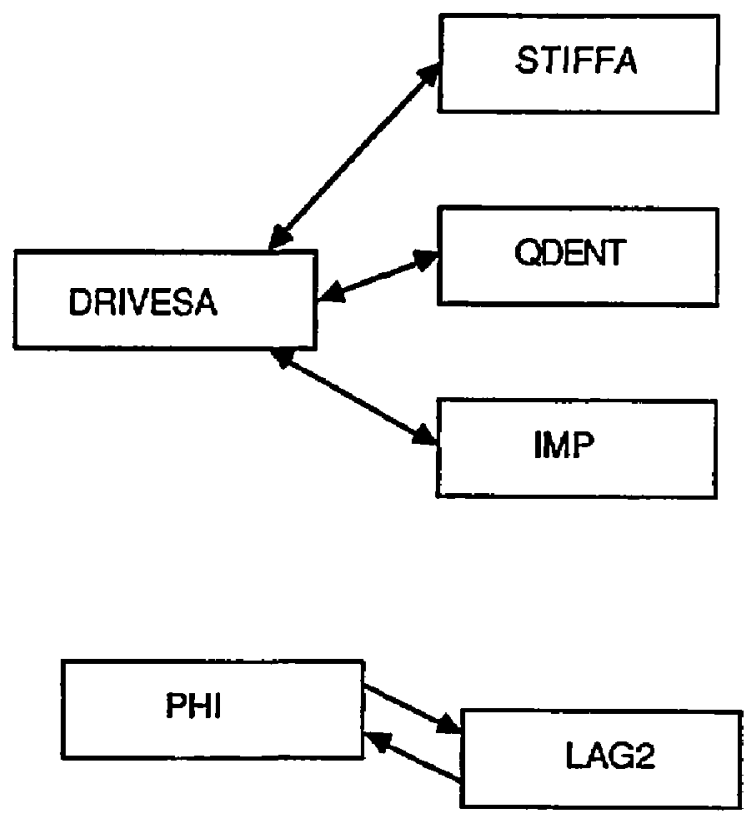

Figure 13: DART subsoutine DRIVESA and function PHI call their own subroutines. 
POT1 solves Laplace's equation in two dimensions

$$
\nabla^{2} \phi=\frac{\partial^{2} \phi}{\partial x^{2}}+\frac{\partial^{2} \phi}{\partial z^{2}}=0
$$

and the relaxation formula is derived by using the central differences

$$
\begin{aligned}
& \frac{\partial^{2} \phi}{\partial x^{2}}=\frac{\phi_{i+1, k}-2 \phi_{i, k}+\phi_{i-1, k}}{(\Delta x)^{2}} \\
& \frac{\partial^{2} \phi}{\partial z^{2}}=\frac{\phi_{i, k+1}-2 \phi_{i, k}+\phi_{i, k-1}}{(\Delta z)^{2}}
\end{aligned}
$$

Substituting these into Eq. 9 gives the relaxation formula:

$$
\phi_{i, k}=\frac{\phi_{i+1, k}+\phi_{i-1, k}+\left(\frac{\Delta_{\varepsilon}}{\Delta x}\right)^{2}\left(\phi_{i, k+1}+\phi_{i, k-1}\right)}{2\left[1+\left(\frac{\Delta z}{\Delta x}\right)^{2}\right]}
$$

Relaxation involves iterating Eq. 12. The rate of convergence is increased by overrelaxation as defined by

$$
\phi_{i, k}^{n}=\phi_{i, k}^{n-1}+\alpha\left(\phi_{i, k}-\phi_{i, k}^{n-1}\right) \text {, }
$$

where $\phi_{i, k}$ is the result from Eq. $12, n$ is the iteration number, and $\alpha>1$ (typically $\alpha \approx 1.8$ ). The file DARTOUT contains displayed selected values of $\phi$ at various points to check convergence.

\subsection{POT}

The potential subroutine is called POT. On its first call, POT calls GETGRID to set up where the electrodes. If isc $=0$, it calls POT1 to get the vacuum potential with this configuration. POT uses a relaxation technique to calculate space charge potentials due to charge densities calculated in QDENT and the electron distribution described in Section 7.

To calculate the space charge potential, we must solve Poisson's equation,

$$
\nabla^{2} \phi=\frac{\partial^{2} \phi}{\partial x^{2}}+\frac{\partial^{2} \phi}{\partial z^{2}}=-\frac{\rho}{\epsilon_{0}} .
$$

The relaxation algorithm derivation is analogous to the derivation shown in the last section and is given by

$$
\phi_{i, k}=\frac{\frac{(\Delta x)^{2}}{\epsilon_{0}} g_{i, k}+\phi_{i+1, k}+\phi_{i-1, k}+\left(\frac{\Delta x}{\Delta z}\right)^{2}\left(\phi_{i, k+1}+\phi_{i, k-1}\right)}{2\left[1+\left(\frac{\Delta z}{\Delta z}\right)^{2}\right]}
$$

where $\epsilon_{0}$ is the dielectric constant of free space and $g_{i, k}$ is the net charge Jensity. $g_{i, k}$ is set to zero if the electron contribution should on tweigh the positive ion contribution.

In order to reduce the sensitivity of the values in the charge density table to details of particular trajectories, a sum of the charge density laid down by all trajectories at each point is used. A description of DART's snapshot technique for generating the charge density table is found in Section 6.10. 


\subsection{START}

START generates the starting conditions for DART. It initializes particle positions, energies, and currents. The currents may be distributed among trajectories with a Gaussian profile. If $z 0(i)=0$ for the $i$ th set of particles, a uniform distribution is used instead.

The distribution function for a Gaussian profile is

$$
f(z)=\exp \left\{-\left(\frac{x-z_{1}}{z_{0}}\right)^{2}\right\},
$$

where $z_{0}$ and $z_{1}$ are input in the arrays $z 0(i)$ and $z 1(i)$, with the index $i$ corresponding to a particular beam component.

DART calculates the current density by dividing the total current (for the half geometry) given by tcrnt( $i)$ by the area under the curve of $f(z)$ over the beam width, bmw(i). The current assigned to a given trajectory is this current density times $f(z)$ times the spacing between initial positions of the particles. The spacing is determined by the input parameters, ntraj(i) and $b m w(i)$ (see Fig. 4).

\subsection{The ODE Solver}

The ODE solver found in DART is a predecessor of LSODE (the Livermore Solver for Ordinary Differential Equations) [6]. Both were written at LLNL by A. Hindmarsh and use the Adams method for solving a system of first-order ordinary differential equations. The subroutines used by DART are DRTVESA, STIFFA, COSETA, and FUNCT, all documented by comment statements in the dartl listing. A brief description of what they do and how they were adapted for DART follows.

\subsubsection{DRIVESA, STIFFA, COSETTA, and FUNCT}

DRIVESA is the driver subroutine for STIFFA. Within DRIVESA, after STIFFA has been called, plotting data are stored, subroutine QDENT is called to generate charge density tables, subroutine IMP is called to check for impacts, reflection at symmetry planes is accomplished (when isymp $(i)=1$ ), time is checked, and appropriate error messages are printed when problems are encountered.

STIFFA does one step of the integration and uses COSETA to obtain coefficients. It also optimizes the step size.

FUNCT contains the functions that determine the derivative of the integration variables. In other words, it gives $F(Y, t)$ in the equation

$$
\frac{d Y}{d t}=F(Y, t)
$$

where $Y$ is the vector valued function given in Eq. 3 . In DART, this is where velocities and forces are calculated from the electromagnetic fields. $F(Y, t)$ is given in Eq. 4. 


\subsection{PHI and LAG2}

$\mathrm{PHI}$ is the subroutine that gets the potential at a given location in $x$ and $z$. PHI calls on LAG2 to interpolate the table established by POT. LAG2 is a standard interpolator, and the degree of the polynomial used for interpolation is set with the parameter ne in the source file, dartl. The present version of DART is set to interpolate with second-degree polynomials. Again, to change this requires editing the source file. When a boundary is encountered, the interpolator uses the last ne +1 points up to the boundary.

\subsection{PARPLT}

PARPLT produces the picture of the particle trajectories superimposed on the electrode configuration. The example shown in Fig. 7 was produced by DART with the default input parameters. The potential value of a given electrode is printed one grid-point down and to the right of the upper left-hand comer of an electrode. Cold ion trajectories are printed with dashed lines, and secondary electron trajectories are printed with dotted lines.

\subsection{SECPAR}

SECPAR is the secondary electron subroutine and is called if the switch ispar $=1$. In subroutine SECPAR, a secondary electron trajectory is started where an ion impacts an electrode. The trajectory is initially directed back along the path of the incoming ion. Each trajectory carries a current equal to $\gamma$ times the current of the ion trajectory, where $\gamma$ is the secondary electron emission coefficient calculated in subroutine GAMMA. A detailed description of $\gamma$ is given in Section 5.6. SECPAR is called for each beam component separately. If $i$ cold $=1$, secondary electron trajectories are calculated from cold ion impacts when SECPAR is called from CIPROD.

\subsection{CIPROD}

CIPROD uses the production rate table called cipror to determine starting conditions for cold ion trajectories. It is called only when icold $=1$. The maximum number of cold ion trajectories to be followed is determined by nctr. When an element of cipror is greater than the input parameter, crpror, a trajectory is set to start at that location. If the number of starting points exceeds netr, then every other starting location is used. If there are still more starting locations than nctr, then every third location is used, and so on, until the number is less than netr. The current assigned to each cold ion trajectory is given by the number of positions skipped plus one, multiplied by the area of a grid cell and by the cold ion production rate.

The production rate table is calculated in subroutine QDENT simultaneously with the charge density table. Then the ion trajectory velocity and the charge density can be used directly. The production rate formula is

$$
\operatorname{cipror}(i, k)=n_{0} \sigma v \rho,
$$

where $n_{0}$ is the background density determined from the gas pressure input parameter, press, and the conversion factor, $3.5 \times 10^{22} /$ Torr $^{-1} \mathrm{~m}^{-3} ; \sigma$ is the cross section for cold ions 
calculated in subroutine SIGMA; $v$ is the velocity of beam ion trajectories; and $\rho$ is the charge density.

SIGMA is the subroutine containing ionization and charge exchange cross sections for cold ton production. These cross sections depend on the local velocity of beam ions and on the ion and gas species. The identifier, id, is an input parameter and assumes the following values:

- id = $1: \mathrm{He}^{+}$ions in $\mathrm{He}$

- id = $2: \mathrm{He}^{+}$ions in $\mathrm{H}_{2}$

- id $=3: \mathrm{H}^{+}$ions in $\mathrm{H}_{2}, \mathrm{D}^{+}$ions in $\mathrm{D}_{2}$, or $\mathrm{T}^{+}$ions in $\mathrm{T}_{2}$

For default cases, no space charge is accumulated from cold ions. If cold ion space charge is important, set icse $=1$. Then, QDENT will be called for cold ions as well.

\subsection{QDENT}

QDENT generates the charge density table used by POT. After a snapshot interval ssdt, the previous charge density variables are linearly interpolated to determine a new one. Charge is then distributed along the mesh points in a scheme devised by C.K. Birdsall [5]. The following description is taken from the original DART document [1]. References in that document indicate sources of further information.

Ir a given interval of time, $\Delta t$, the charge density contibution of a given particle is

$$
q_{d}=I_{j} \Delta t
$$

$\Delta t$ is the "snapshot" time. If all ions at a given energy are launched at the same time, then at a time $\Delta t$ later, the charge densities are recorded, and again at a time $\Delta t$ later, etc. These pictures may be superimposed to get a table of charge densities. For this to work, the number of particles must be great and the snapshot time must be small enough to get a smooth density distribution. To keep $\Delta t$ small without changing the step size of the integration, DART linearly interpolates between points of a trajectory. The particle location is calculated by interpolation and the charge density is distributed to the four surrounding mesh points to construct the charge density table.

The Birdsall scheme is given by

$$
\begin{aligned}
\rho_{i, k} & =\rho\left[\left(x_{i+1}-x\right)\left(z_{k+1}-z\right) /(\Delta x \Delta z)\right] \\
\rho_{i+1, k} & =\rho\left[\left(x-x_{i}\right)\left(z_{k+1}-z\right) /(\Delta x \Delta z)\right] \\
\rho_{i+1, k+1} & =\rho\left[\left(x-x_{i}\right)\left(z-z_{k}\right) /(\Delta x \Delta z)\right] \\
\rho_{i, k+1} & =\rho\left[\left(x_{i+1}-x\right)\left(z-z_{k}\right) /(\Delta x \Delta z)\right],
\end{aligned}
$$

where

$$
\rho=\frac{q_{d}}{(\triangle x \triangle z)} .
$$

In Eq. $20, q_{d}$ is the charge density ( $\mathrm{C} / \mathrm{m}$ ) contributed by the particle to the table at each multiple of $\Delta t$. 
The table is cumulative for each energy run specified by the energy(i) array at input. The final table is saved in the disk file QDTAB. When further accumulation of charges is desired (for example, if another beam were added), run DART again with igtab $=1$. The input parameter, beta, can be used to damp out most iteration-to-iteration oscillations. A fraction, 1 - beta, of the previous charge density is added to a fraction, beta, of the present one. Set beta $=1$ for no damping.

Remember that snapshot time must be short enough to record charge density a few times per cell based on the maximum velocity. The actual charge density is independent of snapshot time. When the snapshot time is reduced, more snapshots occur per cell and, to compensate, the charge distributed is less per snapshot.

Also, the snapshot time should be greater than the maximum step size allowed, hmax, to ensure that all snapshot times in a trajectory have charge distributed. QDENT is called when $t$ exceeds $s s t+s s d t$, where sst starts at one time step after the start time, tbeg. Then sst is incremented in intervals of $s s d t$, the snapshot time. QDENT interpolates position to the location at time, $s s t+s s d t$, and distributes charge from this location. If hmax is too large, the timesteps may exceed the snapshot time. Then interpolations will be done backward, and the last snapshots may not take place. If ssdt is less than hmax, a warning message is printed to the terminal.

Geometrically the Birdsall scheme represents weighting the charge density allocated to a corner of a grid cell by the fraction of the total area contained in the opposing quadrant (see Fig. 14).

Weighting charge density for a symmetry plane is handled as follows. In a cell that has the symmetry plane as a border all trajectories contribute twice the normal charge to the points in the symmetry plane. This is due to the neighboring trajectories that are supposed to exist on the other side of this plane. A trajectory along this plane will contribute only its normal share, allowing a beam traveling parallel to the symmetry plane to have one trajectory contained within this plane if desired. Having a trajectory in the symmetry plane is typically avoided by the choice of initial conditions. The width of the beam is divided into $n t r a j(i)$ equal columns. One trajectory is then started down each column (see Fig. 4).

A beam that originates on a boundary has charge density at that boundary set equal to the charge density one grid row inward. This simulates a this beam that originated exterior to our field. When constituents of the beam exit the working field (i.e., wheli srajectories leave the charge density table), we allow them to continue one gria row beyond to get a more accurate charge density contribution at the edge of the table. To start a beam at a fixed potential, we must start trajectories on a fictitious wall or electrode.

In addition to charge density calculations, the cold ion production rate table, cipror, described in Section 6.9, is calculated in subroutine QDENT.

\subsection{IMP}

IMP is the impact subroutine. It determines whether the given particle trajectory has intersected an electrode. If so, it returns to DRIVESA, which terminates the trajectory and records final data. 

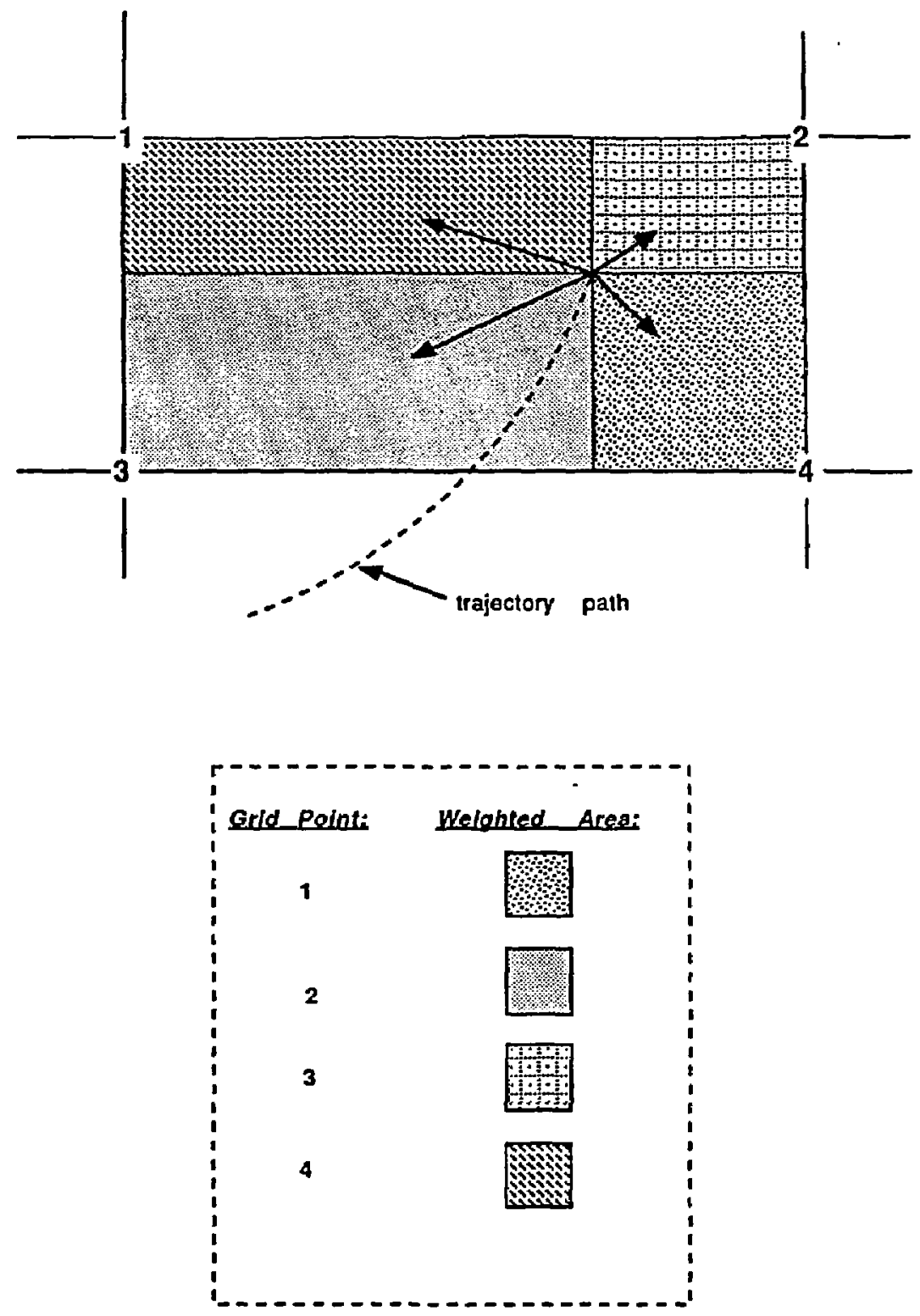

Figure 14: Birdsall's scheme for charge density distribution. The fraction of charge from a snapshot allocated to a comer is determined by the ratio of area in the opposite rectangle to total cell area. 
IMP uses the array called igeom, which consists of ones and zeros, calculated in GETGRID. This array designates the electrodes by values of 1 , interspaced by values of 0 . Then boundaries can be comprised of the diagonals across a given grid cell. A cell with corner values that consist of three ones and a zero is always considered to be a diagonal boundary. Thus, there is no such thing as an inside corner, when avoids confusion.

IMP checks the four corners of a given grid cell for electrode boundaries. If the sum for the four corners is less than three, the given particle has not impacted an electrode.

If the sum is four, a check is performed to determine whether all four points belong to the same elctrode. If they do, an impact will occur. The code now interpolates back along the trajectory path, reduces the step size by a factor of ten, and resumes the trajectory calculation. When the electrode is encountered the code again backs up along the trajectory and reduce the step size. At the third encounter, the position, time, and velority are saved. If the four points do not belong to the same electrode, no impact is recorded and the trajectory calculat6ions continue.

If the sum of the corner values is three, the boundary is diagonal. The code checks to see that all ones belong to the same electrode. If not, there is no impact. If they do, an impact occurs only if the trajectory is in the interior of the triangle with the ones as vertices.

If an impact 6 occurs, the number of the electrode is recorded and the current is adjusted for that electrode. The array called nelec identifies the electrode. This array consists of the numbers zero through numel, and is displayed in DARTOUT and in the graphics output file.

If we have a hit, we record the number of the electrode, and we adjust the collected current on that electrode appropriately. Identification of the electrode is accomplished by checking the array called nelec. This array consists of the numbers, zero through numel, and is displayed in DARTOUT and in the graphics output file.

\section{Modeling Electrons}

When space charge calculations are made and only ions are considered, often a potential builds up that is more positive than a nearby electrode. Such a buildup is nonphysical since the more mobile electrons will prohibit its existence. To model the electron behavior, DART uses two methods chosen by setting the switch called ielmod. No electron calculations are done when $i e l \bmod =0$. When ielmod $=1$, we use a Boltzmann distribution of the form:

$$
q_{c}=A \exp \left\{\frac{\phi-V_{g}}{T_{c}}\right\},
$$

where $V_{g}$ is the reference potential, $T_{e}$ is the electron temperature, and $A$ is given by

$$
A=\mu \bar{q}_{i} \text {. }
$$

$\bar{q}_{i}$ is the average ion charge density taken over a reference area. $\mu$ is $e^{-3}$ and was introduced in this manner apparently to avoid subtracting 3 from the exponent argument repetitively. 
This factor represents the sheath region around an electrode by dropping the potential by a factor of $3 T_{c}$. The electron temperature, $T_{*}$ is the input parameter, te.

If $i e l m o d=2$ we use the reference potentials (plus $3 T_{e}$ ) as upper bounds. If any calculated potential value in a given region is larger, it is set to the upper bound.

This electron modeling is done in three regions: region one is the entry region near the first suppressor in default geometry, and regions two and three are the collector region in default geometry. The boundaries of region one are designated by the dashed line in Fig. 2. In this region, the area for calculating the average ion charge density, $\bar{q}_{i}$, is designated by the input parameters $i l 1$ and $k l 1$, the lower limits, and $i u 1$ and $k u 1$, the upper limits for the $x$ and $z$ grid values in the area calculation. This area is enclosed by the dotted line in Fig. 2 and by the horizontally shaded area at the bottom of Fig. 3. Again, when iel $\bmod =1$, the electrons are modeled by Eq. 21 with reference potential $V_{g}=v g 1$, but only in the region where ine1 $<i<i p e 1$ in $x$ and $k n e 1<k<k p e 1$ in $z$ (region one).

The arguments for electron modeling in regions two and three are analogous. Note that region one may be all or partly included inside region two, and two may be partly included in three. However, the parameter values for the region with highest number prevails in any overlap region.

The electron contribution to the charge density, $q_{00}$, is calculated from the equation:

$$
q_{00}=A \exp \left\{\frac{\phi-V_{g}}{T_{e}}\right\},
$$

and $A$ was defined in Eq. 22. The total charge density is set to zero when the electron charge density exceeds the ion charge density. Oscillations in the charge density from one iteration to another occasionally result when some trajectories are sensitive to small changes in potential. The input parameter, beta, can be used to control most oscillations of this type.

\section{A Beam with Space Charge-Test Case}

As a test case for the DART code, we used a beam (originally uniform) traveling from the left to the right. Space charge calculations cause the beam to diverge. The top wall is fixed at $0.0 \mathrm{kV}$ potential. The current and lengths are chosen to allow a one-dimensional treatment with velocity assumed uniform in $x$ and constant electric field vertical (in $z$ ), which gives a constant acceleration in this direction. The beam thus has a quadratic profile, as shown in Fig. 15.

The input file used to generate these pictures is

$$
\begin{aligned}
& i \min =0 i \max =50 \mathrm{kmin}=0 \mathrm{kmax}=31 \\
& x \max =1 . z \max =.31 \\
& \text { vref }=0 . \\
& \text { Sendn } 11
\end{aligned}
$$


(a)

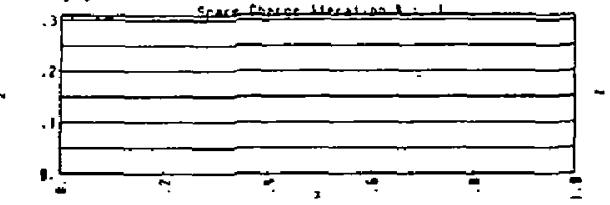

(b)

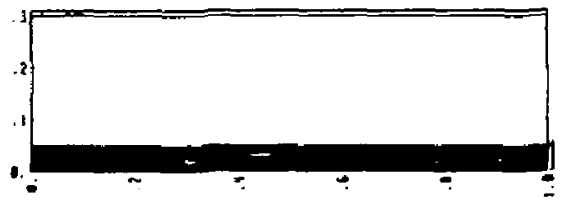

(c)

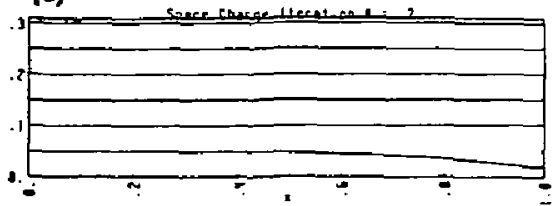

(d)

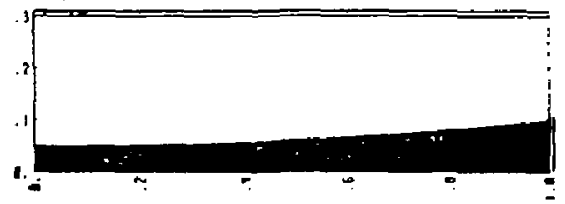

Figure 15: Beam test case showing (a) potential contours, (b) trajectories for vacuum potential, and (c) potential contours, (d) trajectories.

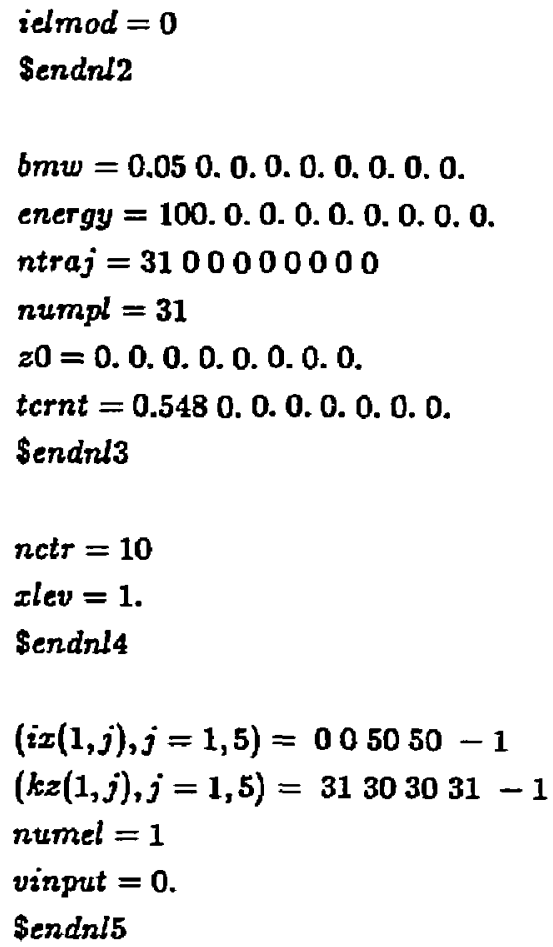

The current is also chosen so the 5-cm beam will double in size to $10 \mathrm{~cm}$ after traveling 
$1 \mathrm{~m}$. This test case was instrumental in improving methods for determining accurate charge density values on the midplane and across the beam to ensure their proper representation.

\section{The Child-Langmuir Law-Test Case}

The Child-Langmuir law is given by the equation

$$
J=\frac{4}{9} \epsilon_{0} \sqrt{2 e / m} \frac{V^{s / 2}}{x^{2}},
$$

where $J$ is the current density $(A / m), V$ is the potential at the object plate $(V) x$ is the distance to the plate $\frac{c}{m}$ is the charge to mass ratio for a species of charge particle and $\epsilon_{0}$ is the free space dielectric constant.

With the DARTIN file given here, the ions produce a space charge potential in agreement with Eq. 24:

$$
\begin{aligned}
& i \min =0 i \max =41 \mathrm{kmin}=0 \mathrm{kmax}=20 \\
& z \max =1.025 . z \max =.5 \\
& \text { vref }=0 \text {. isymp }=1100 \\
& \text { Sendnl1 } \\
& \text { eps } 1=0.005 \text { iternm }=800 \\
& \text { ielmod }=0 \\
& \text { ichl }=1 \\
& \text { Sendril2 } \\
& i z=1 n a=4 \\
& b m w=0.50 .0 .0 .0 .0 .0 .0 .0 \text {. } \\
& \text { energy }=90.050 .0 .0 .0 .0 .0 .0 .0 \text {. } \\
& \text { ntraj }=4000000000 \\
& \text { numpl }=\mathbf{4 0} \\
& z 0=0.0 .0 .0 .0 .0 .0 .0 \text {. } \\
& \text { ternt }=.36750 .0 .0 .0 .0 .0 .0 \text {. } \\
& \text { Sendni3 } \\
& \text { iprint }=1 \\
& \text { Sendnl4 } \\
& (i x(1, j), j=1,5)=40404141-1 \\
& (k z(1, j), j=1,5)=200020-1
\end{aligned}
$$




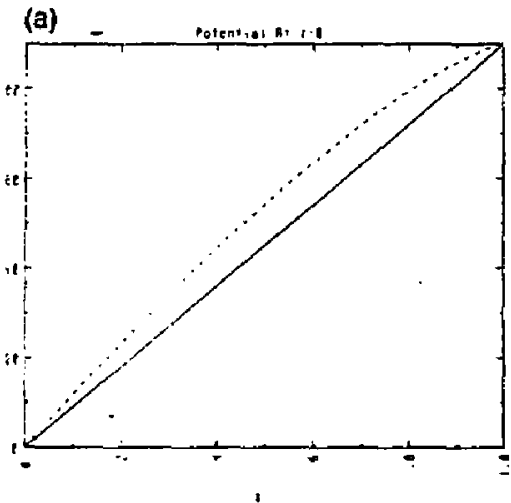

(b)

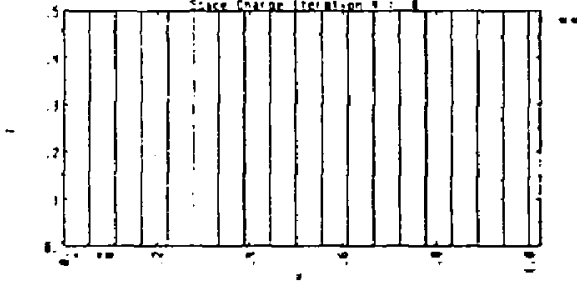

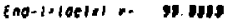

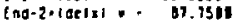

[ng-jidelel : as.sils

(ad-y.laeirl: Bj.j511

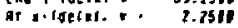

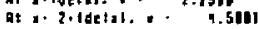

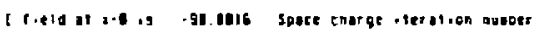

(c)

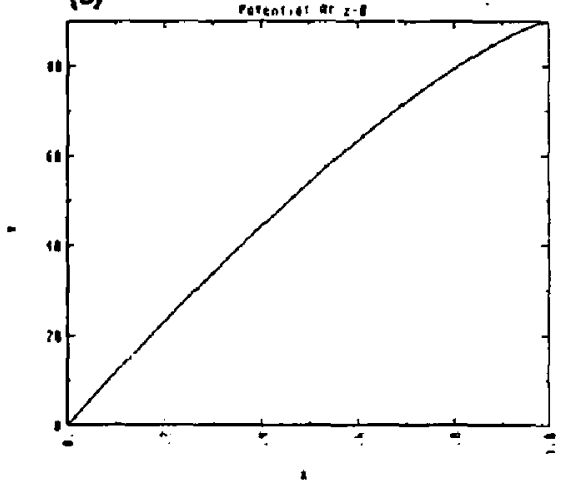

(d)

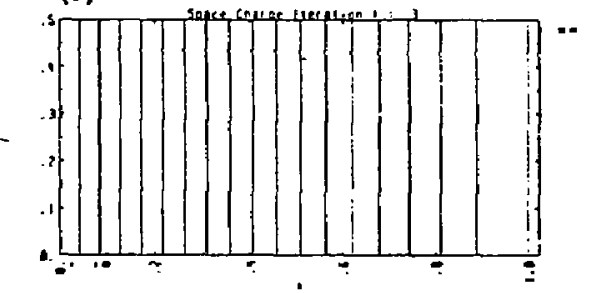

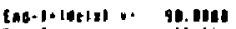

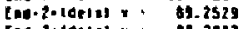

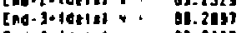

[nd-1-1erlat. 15.9437

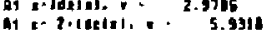

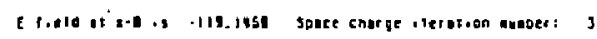

Figure 16: Child-Langmuir law test results. (a) The potential vs $x$ distance for zero space sharge iterations, and (b) potential contours, output potential values at increments of delx from the right-hand side, and the $E$ field at the left-hand side $(x=0)$. (c,d) same as $(a, b)$, respectively after three space charge iterations. 


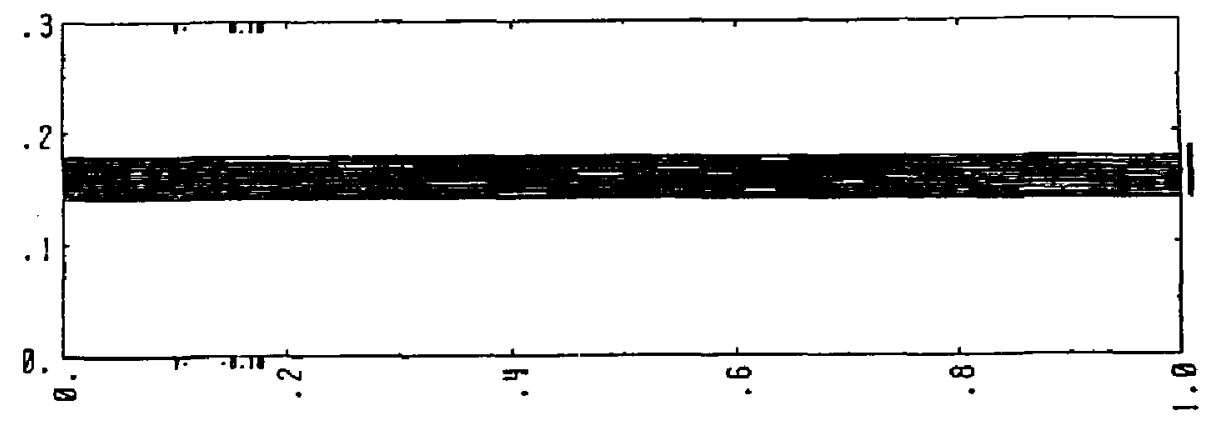

Figure 17: DART output for initial 100-keV ion trajectories.

$$
\begin{aligned}
& (i x(2, j), j=1,3)=00-1 \\
& (k z(2, j), j=1,3)=020-1 \\
& \text { numel }=2 \\
& \text { vinput }=90.0 . \\
& \text { Sendnl5 }
\end{aligned}
$$

This input file was used to generate the results shown in Fig. 16. For this test, we chose a uniform beam of 40 trajectories originating at $\mathrm{D} \mathrm{V}$ and impacting an electrode with 90 $\mathrm{kV}$. The initial energy of the beam is $90.05 \mathrm{keV}$, and the initial current is $0.3675 \mathrm{~A} / \mathrm{m}$. The patential as a function of distance is plotted, with the solid line representing the DART result for the given number of iterations and the dotted line the exact analytic result.

Note The switch ichl should only be set to 1 for the Child-Langmuir law test because special graphs are generated (shown in Fig. 16) that are particular to this test case.

\section{A Test Case for Cold Ion Calculations}

Here we describe a simple test case to check the cold ion production calculations. This test consists of two electrodes with potentials of $\pm 100 \mathrm{~V}$. A $4-\mathrm{cm}$ beam of $100-\mathrm{keV} \mathrm{He}^{+}$ ions passes between the electrodes from left to right through a background hydrogen gas. The generated $0.1-\mathrm{eV}$ cold ions then fall to the negatively charged lower plate, where their current is collected.

The input file is as follows:

$$
\begin{aligned}
& i \min =0 i \max =10 \mathrm{kmin}=0 \mathrm{kmax}=32 \\
& x \max =1 . z \max =.32
\end{aligned}
$$




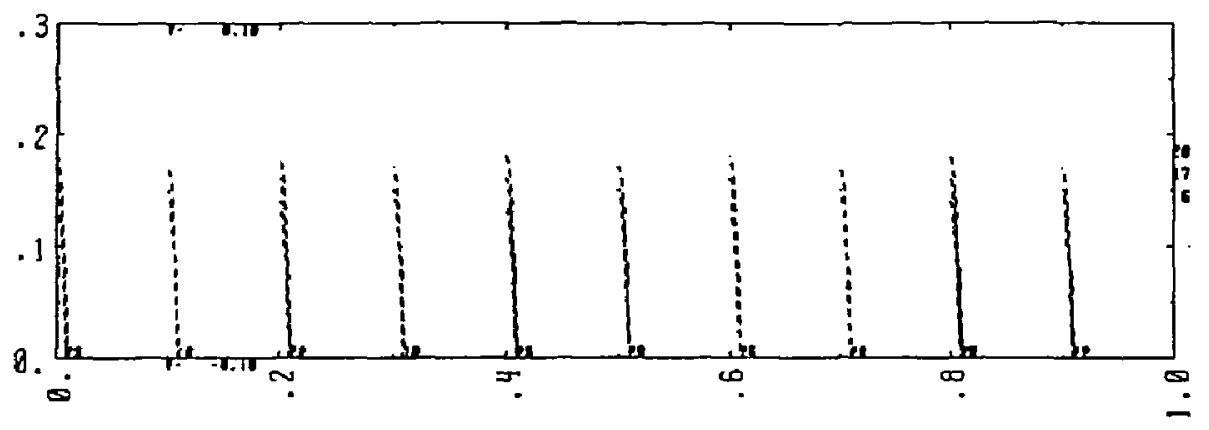

\begin{tabular}{|c|c|c|c|c|c|c|}
\hline $\begin{array}{r}n 0: \\
2 \\
3 \\
5 \\
5 \\
7 \\
8 \\
9 \\
10 \\
11 \\
12 \\
13 \\
14 \\
15 \\
16 \\
17 \\
18 \\
19 \\
21 \\
21 \\
22 \\
23 \\
24 \\
25 \\
28 \\
27 \\
28\end{array}$ & 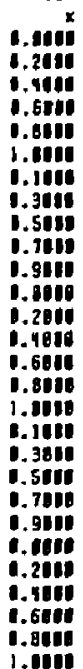 & 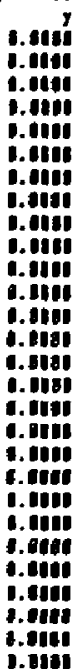 & 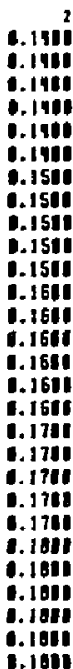 & $\begin{array}{l}31 \\
1 \\
1 \\
1 \\
1 \\
1 \\
1 \\
1 \\
1 \\
1 \\
1 \\
1 \\
1 \\
1 \\
1 \\
1 \\
1 \\
1\end{array}$ & $\begin{array}{r}10 r-0 \\
-2 \\
-2 \\
-2 \\
-2 \\
-2 \\
1 \\
-2 \\
-2 \\
-2 \\
-2 \\
-2 \\
-2 \\
-2 \\
-2 \\
-2 \\
-2 \\
1 \\
-2 \\
-2 \\
-2 \\
-2 \\
-2 \\
-2 \\
-2 \\
-2 \\
-2 \\
-2 \\
1\end{array}$ & 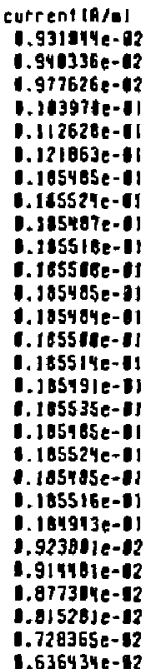 \\
\hline
\end{tabular}

$10191 \cdot 0.488841 \mathrm{et08}$

coliected cold ion currentiafal on electrode 1 . 6.

collecfed coid ion currentla/al on electrode $2 \cdot 0.379937 t+B 2$

Tatal coilected currentihial for this bean cononent on electrode 1 . I.

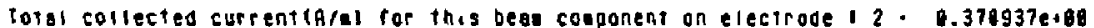

Cold ans vere calculased akipping every J elevents of the production rote table.

Figure 18: DART output for cold ion trajectories. 


$$
\begin{aligned}
& \text { vref }=0 . \text { isymp }=0000 \\
& \text { \$endnl } 1 \\
& \text { ielmod }=0 \\
& \text { Sendnl2 } \\
& z s=.140 .0 .0 .0 .0 .0 .0 .0 . \\
& \text { bmw }=.040 .0 .0 .0 .0 .0 .0 .0 . \\
& \text { energy }=100.0 .0 .0 .0 .0 .0 .0 .0 . \\
& \text { ntraj }=1600000000 \\
& z 0=0.0 .0 .0 .0 .0 .0 .0 . \\
& \text { ternt }=1.00 .0 .0 .0 .0 .0 .0 . \\
& \text { Sendnl3 } \\
& \text { netr }=50 \\
& \text { icold }=1 \\
& \text { itmax }=0 x l e v=.05 \\
& \text { Sendnl } \\
& (i x(1, j), j=1,5)=010100-1 \\
& (k z(1, j), j=1,5)=31313232-1 \\
& (\text { ix }(2, j), j=1,5)=010100-1 \\
& (k z(2, j), j=1,5)=0011-1 \\
& \text { numel }=2 \\
& \text { vinput }=.1-.1 \\
& \text { \$endnl5 }
\end{aligned}
$$

The electrodes are placed $30 \mathrm{~cm}$ apart, and the beam has a total current of $1.0 \mathrm{~A} / \mathrm{m}$. The background gas pressure is $2 \times 10^{-4}$ Torr, which gives a density of $n_{0}=7 \times 10^{18} \mathrm{~m}^{-3}$. These values yield a cold ion production rate of $9.275 \mathrm{~A} / \mathrm{m}^{3}$ (see Section 6.9).

The current collected at the lower electrode should then be $0.371 \mathrm{~A} / \mathrm{m}$. The results are shown in Figs. 17 and 18. The cold ion energy must generate a velocity. We choose this velocity in the positive $x$ direction. This creates the slight drift of cold ion trajectories to the right.

\section{Default Geometry}

The geometry used in the neutral beams for the Tore-Supra tokamak experiment is briefly described in this section. This geometry was chosen as a default to demonstrate a good 
configuration with a sample of input data and typical design considerations. We also used it as a test to reproduce results obtained by J. Pamèla [3]. The vacuum potential is shown in Fig. 5, the potential with space charge in Fig. 6, and trajectories in Fig. 7.

The electrodes in Fig. 1 are as follows:

1. Part of the neutralizer apparatus. The beam enters at $-100 \mathrm{kV}$.

2. Electron suppressor electrode. Its potential is $-140 \mathrm{kV}$.

3. Ion recovery electrode. Its potential is $-10 \mathrm{kV}$.

4. Walls of the energy recovery system. They are grounded at $0 \mathrm{kV}$.

5. Starting potential for the beam. This is $-100 \mathrm{kV}$.

Figure 2 shows the regions used for electron modeling. In region one, the Bolzmann distribution is referenced to $-100 \mathrm{kV}$. Elsewhere (region two), we reference to ground.

Potential contours for this default geometry are displayed in Figs. 5 and 6, and the ion trajectories subject to these potentials are shown in Fig. 7.

To correctly model this system, we can iterate space charge effects to convergence, we can include ionization and charge exchange due to cold ions, and we can include secondary electrons. We are currently performing more detailed studies with all of these considerations.

\section{Summary}

DART is nonrelativistic and does not presently include any method for defining a magnetic field, although the equations allow for a magnetic field if one is specified. All three coordinates are calculated, but one $(y)$ is ignored except when a magnetic field is present and the third component of velocity is needed. DART could therefore be easily be modified to include a magnetic field by using either formulas or a table of magnetic field data.

The greatest asset of the present version is its geometry independence. An arbitrary electrode configuration with arbitrary starting conditions is easily represented by this code. We believe this new DART will be a useful tool for many applications.

\section{Acknowledgements:}

We would like to thank J. Paméla for supplying the geometry for the default test case, and Peter Willmann for his help with memory and timing studies.

\section{References}

(1) B. C. Howard, W. L. Barr, and R. W. Moir, DART: A Simulation Code for a Direct Energy Converter for Fusion Reactors, UCRL-51557, Lawrence Livermore National Laboratory (1974). 
[2] Peter A. Willmann, Grafl III: User Oriented Science Graphics, M-208, Lawrence Livermore National Laboratory (1987).

[3] J. Paméla, "Preliminary Results on the Design Stuḍy of the Energy Recovery Prototype System for the Tore-Supra Neutral Injectors," Note Technique TS 80-8604, Caderache, France (1986).

[4] C.F. Barnett et al., Atomic Data for Controlled Fusion Research, ORNL-5206, Vol. I, Oak Ridge National Laboratory (1977).

[5] C.K. Birdsall and D. Fuss, J. Comp. Phys., 3, 494 (1969).

[6] A.C. Hindmarsh, in Scientific Computing, R.S. Stepleman, Ed., (North Holland, Amsterdam, 1983).

[7] L.S. Hall, Nuc. Fusion 17, 4, 681 (1977). 